\title{
HARISH-CHANDRA PAIRS FOR ALGEBRAIC AFFINE SUPERGROUP SCHEMES OVER AN ARBITRARY FIELD
}

\author{
AKIRA MASUOKA
}

\begin{abstract}
Over an arbitrary field of characteristic $\neq 2$, we define the notion of Harish-Chandra pairs, and prove that the category of those pairs is anti-equivalent to the category of algebraic affine supergroup schemes. The result is applied to characterize some classes of affine supergroup schemes such as those which are (a) simply connected, (b) unipotent or (c) linearly reductive in positive characteristic.
\end{abstract}

KEY Words: affine supergroup scheme, Hopf superalgebra, Harish-Chandra pair.

Mathematics Subject Classification (2010): 14M30, 16T05, 16W55.

\section{INTRODUCTION}

What is an algebraic group? From the functorial view-point, it is defined as an affine group scheme over a fixed field, say $\mathbb{k}$, to be a representable group-valued functor defined on the category of commutative algebras over $\mathbb{k}$; see [10. If $\mathbb{k}$ is an algebraically closed field of characteristic zero, an algebraic affine group scheme is the same as a linear algebraic group. Every affine group scheme is represented uniquely by a commutative Hopf algebra. Since affine group schemes thus correspond precisely to commutative Hopf algebras, they can be studied by Hopf-algebraic methods; see Hochschild [11], Takeuchi [20], 21], 22]. Especially, Takeuchi made substantial contributions, which are free of characteristic of $\mathbb{k}$, replacing Lie algebras in zero characteristic case with hyperalgebras.

The symmetric tensor category of vector spaces is generalized by the category of super-vector spaces, that is, $\mathbb{Z}_{2}$-graded vector spaces, which has the familiar tensor product and the super-symmetry; see (2.1). Each object, such as ordinary, Hopf or Lie algebras, defined in the former category is generalized by a super-object, such as (Hopf or Lie) superalgebras, defined in the latter category.

What is an algebraic supergroup? It is now automatic to answer this question; one has only to replace commutative algebras with the corresponding super-object. An affine supergroup scheme over a field $\mathbb{k}$ is thus defined to be a representable group-valued functor defined on the category of supercommutative superalgebras over $\mathbb{k}$. Here and in what follows we pose the natural assumption char $\mathbb{k} \neq 2$. An affine supergroup scheme $G$ is represented uniquely by a super-commutative Hopf superalgebra, say $A$, and is written as $G=\operatorname{SSp} A$. $G$ is said to be algebraic if $A$ is affine, that is, finitely generated. See [17, [28], 29] for recent characteristic-free studies of supergroup schemes. 
The notion of Harish-Chandra pairs was first introduced by Kostant [12]; it is such a pair $(G, L)$ of a linear algebraic (or analytic) group $G$ and a finite-dimensional Lie superalgebra $L$ that satisfies some conditions. Recently, Carmeli and Fioresi [8] proved that there is a natural categoryequivalence between the Harish-Chandra pairs and the algebraic affine supergroup schemes (in our terms), under the assumption that $\mathbb{k}$ is an algebraically closed field of characteristic zero; in the analytic situation, the same result had been proved by Kostant [12] (see also Koszul [13]) in the real case, and by Vishnyakova [26] in the real and complex cases both. In this paper we generalize the equivalence proved by Carmeli and Fioresi, removing the assumption on $\mathbb{k}$, and apply the result for characteristic-free study of affine supergroup schemes. Just as shown by Takeuchi in the non-super situation, super-cocommutative Hopf superalgebras, and especially irreducible ones (see Definition 2.77) play an important role, in place of Lie superalgebras. An irreducible super-cocommutative Hopf superalgebra is called a hyper-superalgebra.

Here we supply fundamental references, following kind suggestions by a referee. Manin [14] defined for the first time superschemes in the purely algebraic setting, from a geometric view-point; they are defined as a special class of topological spaces given structure sheaves of super-commutative superalgebras. Among other useful monographs we cite especially Berezin [3], Varadarajan [25, and Carmeli, Caston and Fioresi [7]. Deligne and Morgan [9], and Carmeli et al. 7] give modern treatments of the subject from functorial and geometric view-points both, mainly in the differential-geometric setting. Boseck [5], 6] took the functorial (or Hopf-algebraic) view-point in the purely algebraic setting, though the rational points considered were restricted to those in exterior algebras. The notion of hyper-superalgebras is not new. For example, the hyper-superalgebra of the algebraic supergroup $Q(n)$ was thoroughly studied by Brundan and Kleshchev [4].

Let us describe the construction of this paper. After some preliminaries in Section 2, we will start with dualizing the notion of Harish-Chandra pairs. A dual Harish Chandra pair is such a pair $(J, V)$ of a cocommutative Hopf algebra $J$ and a right $J$-module $V$, that is equipped with a bilinear map [ , ] : $V \times V \rightarrow P(J)$ with values in the Lie algebra $P(J)$ of all primitives in $J$, and that satisfies some conditions; see Definition 3.1. Given a Lie superalgebra $L=L_{0} \oplus L_{1}$, then the pair $\left(U\left(L_{0}\right), L_{1}\right)$, equipped with the original bracket [ , ] : $L_{1} \times L_{1} \rightarrow L_{0} \subset P\left(U\left(L_{0}\right)\right)$ restricted to $L_{1}$, is a dual Harish-Chandra pair provided char $\mathbb{k} \neq 3$ (and char $\mathbb{k} \neq 2$, as already assumed). If char $\mathbb{k}=0$, every dual Harish-Chandra pair $(J, V)$ with $J$ irreducible arises uniquely in this way from a Lie superalgebra. Given a dual Harish-Chandra pair $(J, V)$, we construct a super-cocommutative Hopf superalgebra, $H(J, V)$; this equals the universal enveloping superalgebra $U(L)$ if $(J, V)$ arises from a Lie superalgebra $L$ as above. We prove in Theorem 3.9 that $(J, V) \mapsto$ $H(J, V)$ gives a category equivalence from the dual Harish-Chandra pairs DHCP to the super-cocommutative Hopf superalgebras CCHSA; this result was outlined by Takeuchi [24]. 
We will dualize rather faithfully the definition, the construction and the result above. Suppose that $C$ is an affine (that is, finitely generated commutative) Hopf algebra, and $W$ is a finite-dimensional right $C$-comodule. Then the dual vector space $W^{*}$ of $W$ is naturally a right module over the dual cocommutative Hopf algebra $C^{\circ}$ of $C$. The pair $(C, W)$ is called a HarishChandra pair if it is equipped with a bilinear map $W^{*} \times W^{*} \rightarrow P\left(C^{\circ}\right)$ with which $\left(C^{\circ}, W^{*}\right)$ forms a dual Harish-Chandra pair; see Definition 4.11, If $\mathbb{k}$ is an algebraically closed field of characteristic zero, every Harish-Chandra pair $(C, W)$ as just defined arises uniquely from a Harish-Chandra pair $(G, L)$ as defined by Carmeli and Fioresi, and others, where $G=\operatorname{Sp} C(\mathbb{k})$, the linear algebraic group of all rational points in $\mathbb{k}$, and $L=P\left(C^{\circ}\right) \oplus W^{*}$; see Remark 4.12(2). Given a Harish-Chandra pair $(C, W)$ (in our sense), we construct an affine Hopf superalgebra, $A(C, W)$; this construction of ours is different from the corresponding construction given in [8, Sect. 3.3], and has an advantage in our verifying very easily that $A(C, W)$ is a Hopf superalgebra; see the proof of Lemma 4.20, and also Remark 4.10, We prove in Theorem 4.23 that $(C, W) \mapsto A(C, W)$ gives a category equivalence from the Harish-Chandra pairs HCP to the affine Hopf superalgebras AHSA; this last category is anti-isomorphic to the category of algebraic affine supergroup schemes.

Just as for group schemes, short exact sequences play an important role in theory of supergroup schemes. Those sequences of affine supergroup schemes correspond precisely to those sequences of super-commutative Hopf superalgebras. In Sections 5.3, 5.4, we reformulate, in a stronger form, some results from [15, Sections 3,5] on short exact sequences of super-(co)commutative Hopf superalgebras, in terms of (dual) Harish-Chandra pairs.

In Sections 68 the results obtained so far will be applied to characterize three classes of affine supergroup schemes. Theorem 6.3 (resp., Theorem 6.6) characterizes simply connected affine supergroup schemes in characteristic zero (resp., in positive characteristic) in terms of the corresponding Lie superalgebras (resp., hyper-superalgebras); this directly generalizes the corresponding result by Hochschild [11] (resp., by Takeuchi [22]) in the nonsuper situation. Theorem 7.3 states that an affine supergroup scheme $G$ is unipotent if and only if the affine group scheme $G_{\text {res }}$ obtained from $G$ by restricting its domain to the category of commutative algebras is unipotent; this is a very recent unpublished result by A. N. Zubkov, and we will give it a very simple proof. Theorem 8.4 shows that if char $\mathbb{k}>2$, every linearly reductive affine supergroup scheme $G=\operatorname{SSp} A$ is necessarily purely even in the sense that $A$ consists of even elements only, whence by Nagata's Theorem, it is of multiplicative type under the additional assumption that $G$ is algebraic and connected.

We will work with a number of categories. Here is a table of their symbols: 
CCHSA super-cocommutative Hopf superalgebras; (2.11)

HySA hyper-superalgebras; (2.11)

LSA Lie superalgebras; (2.11)

AHSA affine Hopf superalgebras; Definition 2.10

DHCP dual Harish-Chandra pairs; Definition 3.1

iDHCP irreducible dual Harish-Chandra pairs; Definition 3.1

HCP Harish-Chandra pairs; Definition 4.11

cHCP connected Harish-Chandra pairs; Definition 4.11

\section{Preliminaries}

2.1. Throughout we work over a fixed field $\mathbb{k}$ whose characteristic char $\mathbb{k} \neq$ 2. In particular the unadorned $\otimes$ denotes the tensor product over $\mathbb{k}$.

A super-vector space is a vector space $V=V_{0} \oplus V_{1}$ graded by $\mathbb{Z}_{2}=\{0,1\}$. Given a homogeneous element $v \in V$, we let $|v| \in \mathbb{Z}_{2}$ denote its parity. The super-vector spaces, $V, W, \ldots$, form a symmetric tensor category with respect to the familiar tensor product $V \otimes W$, and the super-symmetry

$$
c_{V, W}: V \otimes W \stackrel{\simeq}{\longrightarrow} W \otimes V, \quad c_{V, W}(v \otimes w)=(-1)^{|v||w|} w \otimes v .
$$

We call objects, such as algebra or Hopf-algebra objects, in this symmetric tensor category, attaching 'super' to their original names, so as superalgebras or Hopf superalgebras. They are said to be purely even (resp., purely odd), if they consist of even (resp., odd) elements only. To distinguish the tensor products of super(co)algebras, on which the super-symmetry does effect, from those of non-super ones, we will write $A \otimes B$ for $A \otimes B$.

Given a vector space $V$, we let $V^{*}$ denote the dual vector space. This is a super-vector space so that $\left(V^{*}\right)_{i}=\left(V_{i}\right)^{*}, i=0,1$, if $V$ is. By a pairing of super-vector spaces $V, W$, we mean a bilinear form $\langle\rangle:, V \times W \rightarrow \mathbb{k}$ such that $\left\langle V_{i}, W_{j}\right\rangle=0$ if $i \neq j$ in $\mathbb{Z}_{2}$. A pairing induces linear maps $V \rightarrow W^{*}$, $W \rightarrow V^{*}$ preserving the parity.

Given two pairings $\langle\rangle:, V \times W \rightarrow \mathbb{k},\langle\rangle:, Z \times U \rightarrow \mathbb{k}$ of super-vector spaces, we define their tensor product $\langle\rangle:, V \otimes Z \times W \otimes U \rightarrow \mathbb{k}$ by

$$
\langle v \otimes z, w \otimes u\rangle=\langle v, w\rangle\langle z, u\rangle,
$$

where $v \in V, w \in W, z \in Z, u \in U$.

Lemma 2.1. With the notation as above we have

$$
\left\langle c_{Z, V}(z \otimes v), w \otimes u\right\rangle=\left\langle z \otimes v, c_{U, W}(u \otimes w)\right\rangle .
$$

Proof. We may suppose $|v|=|w|,|z|=|u|$, since otherwise, the both sides are equal to zero. We see then that the both sides are equal to $-\langle v, w\rangle\langle z, u\rangle$ if $|v|=|z|=1$, and to $\langle v, w\rangle\langle z, u\rangle$ in the remaining cases.

2.2. Given a coalgebra $C$, we let $\Delta: C \rightarrow C \otimes C, \varepsilon: C \rightarrow \mathbb{k}$ denote the coproduct and the counit, respectively. To present the coproduct explicitly, we use the Heyneman-Sweedler notation [19, Sect. 1.2] of the form

$$
\Delta(c)=\sum c_{(1)} \otimes c_{(2)}, c \in C .
$$


For a Hopf algebra or superalgebra $A, S: A \rightarrow A$ denotes the antipode, in addition to $\Delta: A \rightarrow A \otimes A, \varepsilon: A \rightarrow \mathbb{k}$ as above. We let

$$
A^{+}=\operatorname{Ker} \varepsilon
$$

denote the augmentation ideal of $A$.

A pairing $\langle\rangle:, H \times A \rightarrow \mathbb{k}$ of Hopf superalgebras $H, A$ is called a $H o p f$ pairing, if we have

$$
\begin{aligned}
& \langle x y, a\rangle=\sum\left\langle x, a_{(1)}\right\rangle\left\langle y, a_{(2)}\right\rangle, \\
& \langle x, a b\rangle=\sum\left\langle x_{(1)}, a\right\rangle\left\langle x_{(2)}, b\right\rangle, \\
& \langle 1, a\rangle=\varepsilon(a), \quad\langle x, 1\rangle=\varepsilon(x),
\end{aligned}
$$

where $x, y \in H, a, b \in A$. It then results that

$$
\langle S(x), a\rangle=\langle x, S(a)\rangle, \quad x \in H, a \in A .
$$

Definition 2.2. We say that a Hopf superalgebra $A$ is $\mathbb{N}$-graded, where $\mathbb{N}=\{0,1,2, \ldots\}$ is the semigroup of all non-negative integers, if $A$ is $\mathbb{N}$ graded, $A=\bigoplus_{n=0}^{\infty} A(n)$, as an algebra and coalgebra, and if the $\mathbb{N}$-grading gives rise to the original $\mathbb{Z}_{2}$-grading so that

$$
A_{0}=\bigoplus_{i \geq 0} A(2 i), \quad A_{1}=\bigoplus_{i \geq 0} A(2 i+1) .
$$

We say that a Hopf pairing $\langle\rangle:, H \times A \rightarrow \mathbb{k}$ of $\mathbb{N}$-graded Hopf superalgebras $H, A$ is $\mathbb{N}$-homogeneous, provided

$$
\langle H(n), A(m)\rangle=0 \text { if } n \neq m \text { in } \mathbb{N} .
$$

A typical example of $\mathbb{N}$-graded Hopf superalgebras is the exterior algebra $\wedge(V)$ of a vector space $V$, which is given the canonical $\mathbb{N}$-grading, and in which every element $v$ in $V$ is primitive [19, p.199], that is, $\Delta(v)=1 \otimes v+$ $v \otimes 1$. Suppose $\operatorname{dim} V<\infty$. Then the canonical pairing $\langle\rangle:, V \times V^{*} \rightarrow \mathbb{k}$ extends uniquely to an $\mathbb{N}$-homogeneous Hopf pairing $\langle\rangle:, \wedge(V) \times \wedge\left(V^{*}\right) \rightarrow$ $\mathbb{k}$, which is determined by

$$
\left\langle v_{1} \wedge \cdots \wedge v_{n}, w_{1} \wedge \cdots \wedge w_{n}\right\rangle=\sum_{\sigma \in \mathfrak{S}_{n}} \operatorname{sgn} \sigma\left\langle v_{1}, w_{\sigma(1)}\right\rangle \ldots\left\langle v_{n}, w_{\sigma(n)}\right\rangle,
$$

where $v_{i} \in V, w_{i} \in V^{*}, n>0$. Note that this is a non-degenerate pairing; see Remark 2.5 below.

2.3. Suppose that $A=\bigoplus_{n=0}^{\infty} A(n)$ is an $\mathbb{N}$-graded Hopf superalgebra. Set

$$
\widehat{A}=\prod_{n=0}^{\infty} A(n) .
$$

As a superalgebra this is the completion of $A$ with respect to the linear topology defined by the descending chain $I_{n}:=\bigoplus_{i \geq n} A(i), n=0,1, \ldots$, of super-ideals. The complete tensor product $\widehat{A} \widehat{\otimes} \widehat{A}$ is the completion of the tensor product $\widehat{A} \otimes \widehat{A}$ of superalgebras with respect the linear topology defined by the descending chain $\widehat{I}_{n} \otimes \widehat{A}+\widehat{A} \otimes \widehat{I}_{n}, n=0,1, \ldots$, of super-ideals, where we set $\widehat{I}_{n}=\prod_{i \geq n} A(i)$. See [23, Sect. 1.5] for the definition in a more general situation. Regard $\mathbb{k}$ as to be discrete. The structure maps on $A$ 
are completed to $\widehat{\Delta}: \widehat{A} \rightarrow \widehat{A} \widehat{\otimes} \widehat{A}, \widehat{\varepsilon}: \widehat{A} \rightarrow \mathbb{k}, \widehat{S}: \widehat{A} \rightarrow \widehat{A}$, which together satisfy the familiar Hopf-algebra axioms with $\otimes$ replaced by $\widehat{\otimes}$. Therefore, this may be called a complete topological Hopf superalgebra. One sees that $A$ recovers from $\widehat{A}$ as

$$
\operatorname{gr} \widehat{A}:=\bigoplus_{n=0}^{\infty} \widehat{I}_{n} / \widehat{I}_{n+1} .
$$

Note that an $\mathbb{N}$-homogeneous pairing $\langle\rangle:, H \times A \rightarrow \mathbb{k}$ of $\mathbb{N}$-graded Hopf superalgebras $H, A$ extends uniquely to a pairing

$$
\langle,\rangle: H \times \widehat{A} \rightarrow \mathbb{k}
$$

such that for each $x \in H,\langle x,-\rangle: \widehat{A} \rightarrow \mathbb{k}$ is continuous. We see that the extended pairing has the properties which are the same as, or analogous to (2.2), (2.3), (2.4).

2.4. Let $A$ be an algebra. Let $A^{\circ}$ denote the dual coalgebra of $A$; see [19, p.109]. By definition it consists of those elements in $A^{*}$ which annihilate some ideal $I \subset A$ of cofinite dimension, that is, $\operatorname{dim} A / I<\infty$. It follows that $A^{\circ}$ is the directed union $\bigcup_{I}(A / I)^{*}$ of the finite-dimensional coalgebras $(A / I)^{*}$

Lemma 2.3. Suppose that $A$ is a superalgebra. Then $A^{\circ}$ consists of those elements in $A^{*}$ which annihilate some super-ideal $I \subset A$ of cofinite dimension. Therefore, $A^{\circ}$ is a super-coalgebra; cf. [15, p.290].

Proof. By [19, Proposition 6.0.3], $A^{\circ}$ coincides with the pullback of $A^{*} \otimes A^{*}$ along the dual map $A^{*} \rightarrow(A \otimes A)^{*}$ of the product on $A$. Since $A^{*} \otimes A^{*}$ is a super-vector subspace of $(A \otimes A)^{*}$, it follows that $A^{\circ}$ is a super-vector subspace of $A^{*}$. The lemma follows, since if a homogeneous element in $A^{*}$ annihilates some ideal $I$ of $A$, then it annihilates the smallest super-ideal of $A$ including $I$.

Corollary 2.4. Suppose that $A$ is a Hopf superalgebra. Equipped with the ordinary dual algebra and coalgebra structures, $A^{\circ}$ forms a Hopf superalgebra. If $A$ is super-commutative (resp., super-cocommutative), then $A^{\circ}$ is super-cocommutative (resp., super-commutative),

Proof. We see from Lemma 2.3 that $A \mapsto A^{\circ}$ gives a contravariant functor from the category of superalgebras to the category of super-coalgebras. Given superalgebras $A, B$, define $\varphi: A^{\circ} \underline{\otimes} B^{\circ} \rightarrow(A \underline{\otimes} B)^{\circ}$ by

$$
\varphi(f \otimes g)(a \otimes b)=f(a) g(b), \quad f \in A^{\circ}, g \in B^{\circ}, a \in A, b \in B .
$$

Slightly modifying the proof of [19, Lemma $6.0 .1 \mathrm{~b})]$, one sees that $\varphi$ is a linear isomorphism, by which we will identify so as $A^{\circ} \otimes A^{\circ}=(A \otimes A)^{\circ}$. Lemma 2.1 implies $\left(c_{A, B}\right)^{\circ}=c_{B^{\circ}, A^{\circ}}$, from which we see that $\varphi$ is an isomorphism of super-coalgebras, and it makes $A \mapsto A^{\circ}$ into a tensor functor preserving the super-symmetry. Just as proving the corresponding result in the non-super situation (see [19, pp.122-123]), we see that if $A$ is a Hopf superalgebra, then $A^{\circ}$ forms a Hopf superalgebra with respect to $\Delta^{\circ}: A^{\circ} \underline{\otimes} A^{\circ}=(A \underline{\otimes} A)^{\circ} \rightarrow A^{\circ}, \varepsilon^{\circ}: \mathbb{k}=\mathbb{k}^{\circ} \rightarrow A^{\circ}, S^{\circ}: A^{\circ} \rightarrow A^{\circ}$. The last assertion is now easy to see. 
Remark 2.5. Looking at the equations (2.2) which define Hopf pairings, one might have felt it strange that the super-symmetry is not involved. But, Corollary 2.4 justifies it. One sees now that a pairing $\langle\rangle:, H \times A \rightarrow \mathbb{k}$ of Hopf superalgebras $H, A$ is a Hopf pairing if and only if $x \mapsto\langle x,-\rangle$ (or $a \mapsto\langle-, a\rangle$ ) gives a Hopf superalgebra map $H \rightarrow A^{\circ}$ (or $A \rightarrow H^{\circ}$ ). For example, the non-degenerate Hopf pairing (2.5) induces an isomorphism $\wedge\left(V^{*}\right) \stackrel{\simeq}{\longrightarrow}(\wedge(V))^{*}$ of $\mathbb{N}$-graded Hopf superalgebras, if $\operatorname{dim} V<\infty$.

2.5. Let $C$ be a super-coalgebra, or in other words, a $\mathbb{Z}_{2}$-graded coalgebra. One can construct the smash (or semi-direct) coproduct $\mathbb{Z}_{2}<C$; this is the coalgebra constructed on the vector space $\mathbb{k} \mathbb{Z}_{2} \otimes C$ with respect to the structure

$$
\Delta(i \otimes c)=\sum\left(i \otimes c_{(1)}\right) \otimes\left(\left(\left|c_{(1)}\right|+i\right) \otimes c_{(2)}\right), \quad \varepsilon(i \otimes c)=\varepsilon(c),
$$

where $i=0,1$ in $\mathbb{Z}_{2}$, and $c \in C$. Note that a $C$-super-comodule is precisely a $\mathbb{Z}_{2}<C$-comodule.

Let Corad $C$ be the coradical, that is, the (direct) sum of all simple subcoalgebras of $C$. Since it is stable under the $\mathbb{Z}_{2}$-action which naturally corresponds to the $\mathbb{Z}_{2}$-grading, Corad $C$ is a super-subcoalgebra, so that $\mathbb{Z}_{2}<$ Corad $C$ is constructed.

Lemma 2.6. We have

$$
\mathbb{Z}_{2} \ltimes \operatorname{Corad} C=\operatorname{Corad}\left(\mathbb{Z}_{2} \ltimes C\right) .
$$

Proof. Since $C$ is a directed union of finite-dimensional super-subcoalgebras, we may and do assume $\operatorname{dim} C<\infty$. Set $R=C^{*}$, the dual algebra of $C$. Let $\operatorname{Rad} R$ be the Jacobson radical of $R$. Then, $(\operatorname{Corad} C)^{*}=R / \operatorname{Rad} R$. On $R, \mathbb{Z}_{2}$ acts as algebra-automorphisms by transposing the $\mathbb{Z}_{2}$-action on $C$, and $\operatorname{Rad} R$ is stable under the action; there arise, therefore, the semi-direct products below. The desired result follows by dualizing the well-known equality

$$
\mathbb{Z}_{2} \ltimes \operatorname{Rad} R=\operatorname{Rad}\left(\mathbb{Z}_{2} \ltimes R\right)
$$

in $\mathbb{Z}_{2} \ltimes R$.

Definition 2.7. A Hopf superalgebra $A$ is said to be irreducible, if Corad $A=$ $\mathbb{k}$; see [19, p.157]. By Lemma [2.6, this is equivalent to saying that the simple $A$-super-comodules are exhausted by the purely even or odd, trivial $A$-comodule $\mathbb{k}$.

Just as in the non-super situation (see [19, Sect. 9.1]), one sees that given a Hopf superalgebra $A$, the largest subcoalgebra $A^{1}$ of $A$ such that Corad $A^{1}=\mathbb{k}$ is an irreducible Hopf super-subalgebra of $A$. We call this $A^{1}$ the irreducible component of $A$ containing 1.

Definition 2.8. A hyper-superalgebra is an irreducible super-cocommutative Hopf superalgebra.

This is the direct generalization of the notion of hyperalgebras 20, which are defined to be irreducible cocommutative Hopf algebras. We let

$$
\text { CCHSA, HySA, LSA }
$$


denote the category of super-cocommutative Hopf superalgebras, the full subcategory consisting of all hyper-superalgebras, and the category of Lie superalgebras, respectively. Given $L \in \mathrm{LSA}$, the universal enveloping superalgebra $U(L)$ uniquely forms a Hopf superalgebra in which every element in $L$ is primitive. Generated by primitives $L$, this $U(L)$ is a hyper-superalgebra; see [19, Exercise 2) on p.224]. Kostant's Theorem states that if char $\mathbb{k}=0$, then $L \mapsto U(L)$ gives a category equivalence

$$
\mathrm{LSA} \approx \mathrm{HySA} .
$$

2.6. The category of super-commutative superalgebras has $\otimes$ as coproduct. A representable group-valued functor $G$ defined on that category is called an affine supergroup scheme, which is represented uniquely by a supercommutative Hopf superalgebra, say $A$, and is denoted by

$$
G=\operatorname{SSp} A .
$$

Therefore, the category of affine supergroup schemes is anti-isomorphic to the category of super-commutative Hopf superalgebras. We say that $G$ is purely even, if $A$ is so, that is, if $A=A_{0}$.

A left (resp., right) rational supermodule over an affine supergroup scheme $G=\operatorname{SSp} A$ is by definition a right (resp., left) $A$-super-comodule.

Definition 2.9. Let $G=\operatorname{SSp} A$ be an affine supergroup scheme.

(1) $G$ is said to be unipotent if the simple rational $G$-supermodules are exhausted by the purely even or odd, trivial $G$-module $\mathbb{k}$, or equivalently if $A$ is irreducible.

(2) $G$ is said to be linearly reductive if every rational $G$-module is semisimple. By Lemma 2.6, this is equivalent to saying that $A$ is cosemisimple, that is, $A=\operatorname{Corad} A$.

An affine supergroup scheme $G=\operatorname{SSp} A$ is said to be algebraic if $A$ is finitely generated.

Definition 2.10. A Hopf superalgebra is said to be affine, if it is supercommutative and finitely generated. We denote by AHSA the category of affine Hopf superalgebras.

The category of algebraic affine supergroup schemes is anti-isomorphic to AHSA.

\section{Super-Cocommutative Hopf superalgebras and Dual HARISH-CHANDRA PAIRS}

3.1. Given a Hopf superalgebra $A$, we let

$$
P(A)=\{u \in A \mid \Delta(u)=1 \otimes u+u \otimes 1\}
$$

denote the super-vector subspace of $A$ consisting of all primitives; this forms a Lie superalgebra with respect to the super-commutator

$$
[u, v]:=u v-(-1)^{|u||v|} v u,
$$

where $u, v$ are homogeneous elements in $P(A)$. This notation may and will soon be used for ordinary Hopf algebras, too. 
Let $J$ be a cocommutative Hopf algebra. Then $P(J)$ is stable under the right adjoint $J$-action

$$
u \mapsto \sum S\left(a_{(1)}\right) u a_{(2)}, \quad u \in P(J), a \in J .
$$

Let $V$ be a right $J$-module. We denote the $J$-action on $V$ by $v \triangleleft a$, where $v \in V, a \in J$.

Definition 3.1. $(J, V)$ is called a dual Harish-Chandra pair, if it is equipped, as its structure, with a bilinear map [, ] : $V \times V \rightarrow P(J)$ with values in the Lie algebra of all primitives in $J$, such that

$$
\begin{aligned}
& \text { (a) } \sum\left[u \triangleleft a_{(1)}, v \triangleleft a_{(2)}\right]=\sum S\left(a_{(1)}\right)[u, v] a_{(2)} \text {, } \\
& \text { (b) }[u, v]=[v, u], \\
& \text { (c) } v \triangleleft[v, v]=0
\end{aligned}
$$

for all $u, v \in V, a \in J$. Note that Condition (c), applied to $u+v+w$ and combined with (b), implies

(d) $u \triangleleft[v, w]+v \triangleleft[w, u]+w \triangleleft[u, v]=0, \quad u, v, w \in V$.

Conversely, (d) implies (c) provided char $\mathbb{k} \neq 3$.

A dual Harish-Chandra pair $(J, V)$ is said to be irreducible, if $J$ is irreducible; see Definition 2.7 .

A morphism $(J, V) \rightarrow\left(J^{\prime}, V^{\prime}\right)$ of dual Harish-Chandra pairs is a pair of a Hopf algebra map $f: J \rightarrow J^{\prime}$ and a linear map $g: V \rightarrow V^{\prime}$ such that

$$
g(v \triangleleft a)=g(v) \triangleleft^{\prime} f(a), \quad f([u, v])=[g(u), g(v)]^{\prime}
$$

for all $u, v \in V, a \in J$. The dual Harish-Chandra pairs and their morphisms naturally form a category DHCP. We let iDHCP denote the full subcategory consisting of all irreducible dual Harish-Chandra pairs.

Remark 3.2. (1) Let $L$ be a Lie algebra. Suppose that $V$ is a right $L$ Lie module, or equivalently a right module over the universal enveloping algebra $U(L)$ of $L$. Given a bilinear map [, ] : $V \times V \rightarrow L$, extend it, as well as the bracket on $L$, to $L \oplus V$, by defining $[v, a]=-[a, v]:=v \triangleleft a$ for $a \in L, v \in V$. Assume char $\mathbb{k} \neq 3$, adding to the original assumption char $\mathbb{k} \neq 2$. Then we see that $(U(L), V)$ together with the given [, ] is a dual Harish-Chandra pair if and only if $L \oplus V$ forms a Lie superalgebra with respect to the extended bracket, in which $L$ is even, and $V$ odd. Indeed, in the definition above, the equations in Conditions (a), (d) are the same as the Jacobi identity, under (b). Thus, every Lie superalgebra $L_{0} \oplus L_{1}$ gives rise to an irreducible dual Harish-Chandra pair $\left(U\left(L_{0}\right), L_{1}\right)$.

(2) Suppose char $\mathbb{k}=0$. By Kostant's Theorem [19, Theorem 13.0.1] (see also (2.12) ), every hyperalgebra (that is, irreducible cocommutative Hopf algebra) $J$ is of the form $U(L)$, where $L=P(J)$. It follows from Part 1 above that every irreducible Harish-Chandra pair arises uniquely from a Lie superalgebra.

(3) Suppose char $\mathbb{k}=0$. Let $J$ be a cocommutative Hopf algebra. Suppose that $J$ is pointed [19, p.157]; this necessarily holds if $\mathbb{k}$ is algebraically closed. Set $L=P(J)$, and let $G$ denote the group of all grouplikes in $J$. Again by Kostant's Theorem [19, Theorem 8.1.5], $J=\mathbb{k} G \ltimes U(L)$, the smash (or semi-direct) product of $U(L)$ by the group algebra $\mathbb{k} G$. In particular, $L$ is stable under the adjoint $G$-action $a \mapsto a^{g}:=g^{-1} a g$, where $a \in L, g \in G$. 
Suppose that we have an irreducible dual Harish-Chandra pair $(U(L), V)$; it arises uniquely from a Lie superalgebra structure on $L \oplus V$, as was just seen. We see that $(J, V)$ together with the restricted bracket [, ] $V \times V \rightarrow L$ on the Lie superalgebra $L \oplus V$ turns into a dual Harish-Chandra pair if and only if $V$ is a right $\mathbb{k} G$-module such that $[v, a] \triangleleft g=\left[v \triangleleft g, a^{g}\right]$, where $v \in V, a \in L, g \in G$, and [ , ] $: V \times V \rightarrow L$ is $G$-equivariant, where $G$ acts on $L$ by the adjoint action.

Remark 3.2 (2) shows the following.

Proposition 3.3. If char $\mathbb{k}=0$, then $L \mapsto\left(U\left(L_{0}\right), L_{1}\right)$ gives a category equivalence $\mathrm{LSA} \approx \mathrm{iDHCP}$.

Suppose $H \in$ CCHSA. We define $\underline{H}, V_{H}$ as in [15, p.291], as follows:

$$
\underline{H}=\Delta^{-1}\left(H_{0} \otimes H_{0}\right), \quad V_{H}=P(H)_{1} .
$$

Thus, $V_{H}$ consists of all odd primitives in $H$, and $\underline{H}$ is seen to be the largest ordinary subcoalgebra of $H$; it is indeed a Hopf subalgebra. One sees that the adjoint action

$$
v \triangleleft a=\sum S\left(a_{(1)}\right) v a_{(2)}, \quad v \in V_{H}, a \in \underline{H}
$$

defines a right $\underline{H}$-module structure on $V_{H}$. The super-commutator (3.1) restricted to $V_{H}$ defines a bilinear form

$$
[,]: V_{H} \times V_{H} \rightarrow P(\underline{H})
$$

with values in $P(\underline{H})$, since the purely even primitives in $H$ constitute $P(\underline{H})$.

Proposition 3.4. $\left(\underline{H}, V_{H}\right)$, given the bilinear map above, is a dual HarishChandra pair. This construction is functorial, so that $H \mapsto\left(\underline{H}, V_{H}\right)$ gives a functor CCHSA $\rightarrow$ DHCP.

Proof. Condition (c) is satisfied, since one sees that $[v, v]=2 v^{2} \in P(\underline{H})$, whence $v \triangleleft[v, v]=v\left(2 v^{2}\right)-\left(2 v^{2}\right) v=0$. The remaining is easy to see.

3.2. We wish to construct a quasi-inverse of the functor just obtained.

Given a vector space $V$, let $T(V)=\bigoplus_{n=0}^{\infty} T^{n}(V)$ denote the tensor algebra on $V$; this is $\mathbb{N}$-graded. The following is a special form of a known result in the braided situation; see [1, Definition 3.2.3], for example.

Lemma 3.5. Let $V$ be a vector space as above. The $\mathbb{N}$-graded algebra $T(V)$ turns uniquely into an $\mathbb{N}$-graded Hopf superalgebra in which every element of $V$ is primitive. This $T(V)$ is super-cocommutative.

Remark 3.6. Let us give an explicit description of the coproduct $\Delta$ : $T(V) \rightarrow T(V) \otimes T(V)$, which is called the shuffle coproduct. It is the sum of linear maps $\bar{\Delta}_{n, i}: T^{n}(V) \rightarrow T^{i}(V) \otimes T^{n-i}(V)$, where $0 \leq i \leq n$. Suppose $i=0$ or $n$. Then, $\Delta_{n, 0}: T^{n}(V) \mapsto \mathbb{k} \otimes T^{n}(V)$ and $\Delta_{n, n}: T^{n}(V) \mapsto T^{n}(V) \otimes \mathbb{k}$ are the canonical isomorphisms. Suppose $0<i<n$, and let

$$
\mathfrak{S}_{n, i}=\left\{\sigma \in \mathfrak{S}_{n} \mid \sigma(1)<\cdots<\sigma(i), \sigma(i+1)<\cdots<\sigma(n)\right\}
$$

denote the subset of the symmetric group $\mathfrak{S}_{n}$ of degree $n$ which consists of all $i$-shuffles. Then,

$$
\Delta_{n, i}\left(v_{1} \otimes \cdots \otimes v_{n}\right)=\sum_{\sigma \in \mathfrak{S}_{n, i}} \operatorname{sgn} \sigma\left(v_{\sigma(1)} \otimes \cdots \otimes v_{\sigma(i)}\right) \otimes\left(v_{\sigma(i+1)} \otimes \cdots \otimes v_{\sigma(n)}\right) .
$$


Let $J$ be a cocommutative Hopf algebra, and let $V$ be a right $J$-module. Then $T(V)$ turns naturally into a right $J$-module, with respect the diagonal $J$-action given by

$$
1 \triangleleft a:=\varepsilon(a) 1, \quad\left(v_{1} \otimes \cdots \otimes v_{n}\right) \triangleleft a:=\sum\left(v_{1} \triangleleft a_{(1)}\right) \otimes \cdots \otimes\left(v_{n} \triangleleft a_{(n)}\right),
$$

where $a \in J, v_{i} \in V, 1 \leq i \leq n$.

Lemma 3.7. On $T(V)$, the product $T(V) \underline{\otimes} T(V) \rightarrow T(V)$, the unit $\mathbb{k} \rightarrow$ $T(V)$ and the other structure maps $\Delta: T(V) \rightarrow T(V) \underline{\otimes} T(V), \varepsilon: T(V) \rightarrow$ $\mathbb{k}, S: T(V) \rightarrow T(V)$ are all J-linear, where $\mathbb{k}$ is regarded the trivial right $J$-module via the counit. This means that $T(V)$ is a Hopf-algebra object in the symmetric tensor category $\mathrm{SMod}_{J}$ of right $J$-supermodules.

Proof. We see directly that the product and the unit are $J$-linear. The coproduct $\Delta$ is $J$-linear, since it is an algebra map, and is $J$-linear, restricted to $T^{0}(V) \oplus T^{1}(V)$. Similarly, we see that $\varepsilon$ and $S$ are $J$-linear.

Let $J$ be a cocommutative Hopf algebra, and let $V$ be a right $J$-module. Since $T(V)$ is in particular an algebra object in $\operatorname{SMod}_{J}$ by Lemma 3.7, we can construct the algebra

$$
\mathcal{H}(J, V):=J \ltimes T(V)
$$

of smash (or semi-direct) product; see [19, Sect. 7.2]. This is the tensor product $J \otimes T(V)$ as a vector space, and is an $\mathbb{N}$-graded algebra with $\mathcal{H}(J, V)(n):=J \otimes T^{n}(V), n \in \mathbb{N}$. The cocommutativity of $J$ ensures the following, just as in the non-super situation.

Proposition 3.8. $\mathcal{H}(J, V)$ turns uniquely into an $\mathbb{N}$-graded Hopf superalgebra which includes $J=J \otimes \mathbb{k}, T(V)=\mathbb{k} \otimes T(V)$ as Hopf super-subalgebras. This $\mathcal{H}(J, V)$ is super-cocommutative, and the antipode is given by

$$
S(a \otimes x)=(1 \otimes S(x))(S(a) \otimes 1)=\sum S\left(a_{(1)}\right) \otimes\left(S(x) \triangleleft S\left(a_{(2)}\right)\right),
$$

where $a \in J, x \in T(V)$

The following result was outlined by M. Takeuchi [24].

Theorem 3.9. (1) Given $(J, V) \in \mathrm{DHCP}$, let $I(J, V)$ be the two-sided ideal of $\mathcal{H}(J, V)$ generated by all $1 \otimes(u v+v u)-[u, v] \otimes 1$, where $u, v \in V$. Then this is a Hopf super-ideal. Define

$$
H(J, V):=\mathcal{H}(J, V) / I(J, V) .
$$

Then this is a super-cocommutative Hopf superalgebra. This construction is functorial, so that we have the functor

$$
H: \mathrm{DHCP} \rightarrow \mathrm{CCHSA}, \quad(J, V) \mapsto H(J, V) .
$$

(2) The functor $H$ above is an equivalence which has as a quasi-inverse the functor $H \mapsto\left(\underline{H}, V_{H}\right)$ given by Proposition 3.4.

Proof. Here we only prove Part 1 and defer the proof of Part 2 to Section 3.3. Since $1 \otimes(u v+v u)-[u, v] \otimes 1$ is even primitive, it follows that $I(J, V)$ is a Hopf super-ideal. The functoriality is easy to see. 
Remark 3.10. We see from Proposition 3.3 that if char $\mathbb{k}=0$, the equivalence above, restricted to iDHCP, can be identified with the category equivalence LSA $\approx$ HySA given in (2.12).

3.3. To prove the remaining Part 2 above, let $(J, V) \in$ DHCP. Then $H(J, V)$ is in particular an algebra given a natural algebra map from $J$; such an algebra is called a $J$-ring in [2, p.195]. It is naturally a left (and right) $J$-module. Given an element $v \in V$, we write simply $v$ for the natural image of $1 \otimes v \in \mathcal{H}(J, V)$ in $H(J, V)$.

Lemma 3.11. $H(J, V)$ is free as a left $J$-module. In fact, if we choose arbitrarily a basis $X$ of $V$ given a total order, then the products

$$
x_{1} x_{2} \ldots x_{n}, \quad x_{i} \in X, x_{1}<x_{2}<\cdots<x_{n}, n \geq 0
$$

in $H(J, V)$ constitute a $J$-free basis.

Proof. Suppose that $X$ is a totally ordered basis of $V$. As a $J$-ring, $H(J, V)$ is generated by $X$, and is defined by the relations

(i) $x a=\sum a_{(1)}\left(x \triangleleft a_{(2)}\right), \quad x \in X, a \in J$,

(ii) $x y=-y x+[x, y], \quad x, y \in X, x>y$,

(iii) $x^{2}=\frac{1}{2}[x, x], \quad x \in X$.

Here, Condition (b) of Definition 3.1 allows us to suppose in (ii) that $x>y$. We regard the relations (i)-(iii) as a reduction system [2, p.180],

$$
x a \rightarrow \sum a_{(1)}\left(x \triangleleft a_{(2)}\right), \quad x y \rightarrow-y x+[x, y], \quad x^{2} \rightarrow \frac{1}{2}[x, x] .
$$

Here, we suppose that $x \triangleleft a_{(2)}$ is presented as a left linear combination of elements in $X$.

We wish to apply the opposite-sided version of [2, Proposition 7.1]. For this we first define an order $\preceq$ among words $A, B, \ldots$ in $X$ of a fixed length, as follows: we let $A \preceq B$, if $A$ is a permutation of $B$, and contains the same or a smaller number of mis-ordered pairs as or than $B$. Here, a mis-ordered pair in $A=x_{1} x_{2} \ldots x_{n}$ is a pair $\left(x_{i}, x_{j}\right)$ such that $i<j, x_{i}>x_{j}$. Next, we add to $X$ one element $*$, which is a symbol representing any element in $J$. (To be more precise, $*$ represents the factor $J$ in $J \oplus \bigoplus_{x \in X} \mathbb{Z} x$, just as in [2, Proposition 7.1], $\kappa$ represents $k$ in M.) Let $A=x_{1} x_{2} \ldots x_{n}$ be a word in $X \cup\{*\}$. To this $A$, we associate a sequence $s(A)=\left(\epsilon_{1}, \epsilon_{2}, \ldots, \epsilon_{n}\right)$ of the numbers 0,1 , following the rule that $\epsilon_{i}=0$ if $x_{i}=*$, and $\epsilon_{i}=1$ if $x_{i} \in X$. Let $A^{\prime}$ denote the word in $X$ obtained from $A$ by removing all $*$. Given two words $A, B$ in $X \cup\{*\}$, we let $A \leq B$, if one of the following holds:

- length $A<$ length $B$;

- length $A=$ length $B$, and $s(A)$ precedes $s(B)$ in lexicographical order, where we suppose $0<1$;

- length $A=$ length $B, s(A)=s(B)$ and $A^{\prime} \preceq B^{\prime}$.

Then we see that this $\leq$ defines an order among all words in $X \cup\{*\}$, and satisfies the following assumptions required for the result we wish to apply: it is a semigroup order which satisfies the DCC, and which is consistent with our reduction system in the sense that every reduction changes a word to a sum of smaller words. (The order $\leqslant$ on $\langle X \cup\{\kappa\}\rangle$ given in Proposition 7.1 of [2] is assumed to be induced from a pre-order $\leqslant_{0}$ on $\langle X\rangle$, while our 
order is not induced from such a pre-order. However, the assumption is not necessary, and the proposition, in fact the opposite-sided version, can apply to our situation as is seen from its proof.) Therefore, if we see that the ambiguities which may occur when we reduce the words

(iv) $x y a, \quad x \geq y$ in $X, a \in J$,

(v) $x y z, \quad x \geq y \geq z$ in $X$

are all resolvable, Lemma 3.11 follows since the products given above are precisely the irreducible words, that is, the words in $X$ which do not contain any $x y$ with $x \geq y$ in $X$ as a subword. First, let $x y a$ be a word from (iv) with $x>y$. This is reduced on one hand as

$$
x y a \rightarrow \sum x a_{(1)}\left(y \triangleleft a_{(2)}\right) \rightarrow \sum a_{(1)}\left(x \triangleleft a_{(2)}\right)\left(y \triangleleft a_{(3)}\right),
$$

and on the other hand as

$$
\begin{aligned}
x y a & \rightarrow-y x a+[x, y] a \\
& \rightarrow-\sum a_{(1)}\left(y \triangleleft a_{(2)}\right)\left(x \triangleleft a_{(3)}\right)+\sum a_{(1)}\left[x \triangleleft a_{(2)}, y \triangleleft a_{(3)}\right],
\end{aligned}
$$

where in the last step, we have used that the Hopf algebra axioms for $J$ and Condition (a) of Definition 3.1 give

$$
[x, y] a=\sum a_{(1)} S\left(a_{(2)}\right)[x, y] a_{(3)}=\sum a_{(1)}\left[x \triangleleft a_{(2)}, y \triangleleft a_{(3)}\right] .
$$

The two results are seen to be further reduced to the same element, by using the cocommutativity of $J$. Next, let $x y z$ be a word from (ii) with $x>y>z$. Note $x[y, z] \rightarrow[y, z] x+x \triangleleft[y, z]$, since $[y, z]$ is primitive. Then one sees that $x y z$ is reduced on one hand as

$$
\begin{aligned}
x y z & \rightarrow-x z y+x[y, z] \rightarrow z x y-[x, z] y+[y, z] x+x \triangleleft[y, z] \\
& \rightarrow-z y x+z[x, y]-[x, z] y+[y, z] x+x \triangleleft[y, z] \\
& \rightarrow-z y x+[x, y] z+z \triangleleft[x, y]-[x, z] y+[y, z] x+x \triangleleft[y, z],
\end{aligned}
$$

and on the other hand as

$$
\begin{aligned}
x y z & \rightarrow-y x z+[x, y] z \rightarrow y z x-y[x, z]+[x, y] z \\
& \rightarrow-z y x+[y, z] x-[x, z] y-y \triangleleft[x, z]+[x, y] z .
\end{aligned}
$$

The two results coincide by Conditions (b), (d) of Definition 3.1. We have thus seen that the ambiguities in reducing the words in (iv), (v) are resolvable when $x, y$ and $z$ are distinct. We can similarly prove the resolvability in the remaining cases.

We need the following result from [15].

Proposition 3.12 ([15, Theorem 3.6). Let $H \in \mathrm{CCHSA}$, and choose arbitrarily a basis $X$ of $V_{H}$ given a total order. Then the left $\underline{H}$-linear map $\phi_{X}: \underline{H} \otimes \wedge\left(V_{H}\right) \rightarrow H$ defined by

$$
\phi_{X}\left(1 \otimes\left(x_{1} \wedge x_{2} \wedge \cdots \wedge x_{n}\right)\right)=x_{1} x_{2} \ldots x_{n}
$$

on the $\underline{H}$-free basis

$$
1 \otimes\left(x_{1} \wedge x_{2} \wedge \cdots \wedge x_{n}\right), \quad x_{i} \in X, x_{1}<x_{2}<\cdots<x_{n}, n \geq 0
$$

is a unit-preserving isomorphism of super-coalgebras. 
Proof of Part 2 of Theorem 3.9. We will see that the composites of the two functors are isomorphic to the identity functors.

Let $(J, V) \in \mathrm{DHCP}$, and set $H=H(J, V)$. By Lemma 3.11, $H$ naturally includes $J, V$ so that $J \subset \underline{H}, V \subset V_{H}$. Choose such a totally ordered basis $X$ of $V_{H}$ that extends some totally ordered basis of $V$. Lemma 3.11 shows that the composite $J \otimes \wedge(V) \hookrightarrow \underline{H} \otimes \wedge\left(V_{H}\right) \stackrel{\simeq}{\longrightarrow} H$ of the inclusion with the isomorphism $\phi_{X}$ given by Proposition 3.12 is an isomorphism. It follows that $(J, V)=\left(\bar{H}, V_{H}\right)$, which is seen to be a coincidence as objects in DHCP.

Let $H \in \mathrm{CCHSA}$, and construct $H\left(\underline{H}, V_{H}\right)$. We see that the inclusions $\underline{H} \hookrightarrow H, V_{H} \hookrightarrow H$ uniquely extends to a Hopf superalgebra map $\mathcal{H}\left(\underline{H}, V_{H}\right) \rightarrow H$, which factors through $H\left(\underline{H}, V_{H}\right)$. The resulting map, which we denote by

$$
\alpha_{H}: H\left(\underline{H}, V_{H}\right) \rightarrow H,
$$

is natural in $H$, as is easily seen. Choose a totally ordered basis $X$ of $V_{H}$. Then the isomorphism $\phi_{X}: \underline{H} \otimes \wedge\left(V_{H}\right) \stackrel{\simeq}{\longrightarrow} H$ shows that the left $\underline{H}$-free basis $x_{1} x_{2} \ldots x_{n}$ of $H\left(\underline{H}, V_{H}\right)$ obtained in Lemma 3.11 is mapped by $\alpha_{H}$ to such a basis of $H$. Therefore, $\alpha_{H}$ gives a natural isomorphism.

Remark 3.13. As is easily seen, the obtained equivalence restricts to a category equivalence between iDHCP and HySA.

\section{Affine Hopf superalgebras and Harish-Chandra Pairs}

4.1. We dualize the construction of $\mathcal{H}(J, V)$ given in the preceding section.

Let $W$ be a vector space. Let $T_{c}(W)$ be the tensor coalgebra on $W$. It is, as a vector space, the tensor algebra $T(W)$ so that $T_{c}(W)=\bigoplus_{n=0}^{\infty} T^{n}(W)$, and has the following coalgebra structure: the counit $\varepsilon: T_{c}(W) \rightarrow \mathbb{k}$ is the projection onto the zero-th component, and the coproduct $\Delta: T_{c}(W) \rightarrow$ $T_{c}(W) \otimes T_{c}(W)$ is defined by $\Delta(1)=1 \otimes 1$, and in positive degree $n>0$, by

$$
\begin{aligned}
& \Delta\left(v_{1} \otimes \cdots \otimes v_{n}\right)=1 \otimes\left(v_{1} \otimes \cdots \otimes v_{n}\right)+ \\
& \sum_{0<i<n}\left(v_{1} \otimes \ldots v_{i}\right) \otimes\left(v_{i+1} \otimes \ldots v_{n}\right)+\left(v_{1} \otimes \cdots \otimes v_{n}\right) \otimes 1 .
\end{aligned}
$$

This forms an $\mathbb{N}$-graded Hopf superalgebra with respect to the following shuffle product:

$$
\left(v_{1} \otimes \cdots \otimes v_{i}\right)\left(v_{i+1} \otimes \cdots \otimes v_{n}\right)=\sum_{\tau^{-1} \in \mathfrak{S}_{n, i}}(\operatorname{sgn} \tau) v_{\tau(1)} \otimes \cdots \otimes v_{\tau(n)} .
$$

Here, we emphasize that the inverse $\tau^{-1}$ of the running index $\tau$ is required to be an $i$-shuffle. See [1, Definition 3.2.10]. The original 1 in $T^{0}(W)$ still acts as a unit in $T_{c}(W)$, and $T_{c}(W)$ is super-commutative.

Let $C$ be a commutative Hopf algebra, and let $G=\operatorname{Sp} C$ be the corresponding affine group scheme. Suppose that $W$ is a right $C$-comodule; this is equivalent to saying that $W$ is a rational left $G$-module. We present the comodule structure on $W$, as

$$
w \mapsto \sum w_{(0)} \otimes w_{(1)}, W \rightarrow W \otimes C ;
$$

see [19, p.33]. Note that each component, $T^{n}(W)$, of $T_{c}(W)$, being a tensor product of right $C$-comodules, is naturally a right $C$-comodule, and so their 
direct sum $T_{c}(W)$ is, too. Explicitly, the $C$-comodule structure $T^{n}(W) \rightarrow$ $T^{n}(W) \otimes C$ is given by $1 \mapsto 1 \otimes 1$ when $n=0$, and by

$$
w_{1} \otimes \cdots \otimes w_{n} \mapsto \sum\left(\left(w_{1}\right)_{(0)} \otimes \cdots \otimes\left(w_{n}\right)_{(0)}\right) \otimes\left(w_{1}\right)_{(1)} \cdots\left(w_{n}\right)_{(1)}
$$

when $n>0$. We have the following result, which dualizes Lemma 3.7, and is proved easily in the dual manner.

Lemma 4.1. $T_{c}(W)$ is a commutative Hopf-algebra object in the symmetric tensor category $\mathrm{SMod}^{C}$ of right $C$-super-comodules.

It follows that the smash coproduct constructs the coalgebra

$$
\mathcal{A}(C, W):=C<T_{c}(W) .
$$

This coalgebra is constructed on the vector space $C \otimes T_{c}(W)$ with respect to the structure

$$
\begin{aligned}
\Delta(c \otimes z) & \left.=\sum\left(c_{(1)} \otimes\left(z_{(1)}\right)_{(0)}\right) \otimes\left(\left(z_{(1)}\right)_{(1)} c_{(2)}\right) \otimes z_{(2)}\right), \\
\varepsilon(c \otimes z) & =\varepsilon(c) \varepsilon(z),
\end{aligned}
$$

where $c \in C, z \in T_{c}(W)$; this generalizes (2.9). Regard $\mathcal{A}(C, W)$ as the algebra of the tensor product of $C$ and $T_{c}(W)$. Proposition 3.8 is easily dualized as follows.

Proposition 4.2. $\mathcal{A}(C, W)$ is a super-commutative $\mathbb{N}$-graded Hopf superalgebra whose $n$-th component is $C \otimes T^{n}(W)$. The antipode is given by

$$
S(c \otimes z)=\sum S\left(c z_{(1)}\right) \otimes S\left(z_{(0)}\right), \quad c \in C, z \in T_{c}(W) .
$$

Note from Section 2.3 that the $\mathbb{N}$-graded Hopf superalgebra $\mathcal{A}(C, W)$ has the completion, which we denote by

$$
\widehat{\mathcal{A}}(C, W)=\prod_{n=0}^{\infty} C \otimes T^{n}(W) .
$$

4.2. Let $W$ be a finite-dimensional vector space, and set $V=W^{*}$. We have the two $\mathbb{N}$-graded Hopf superalgebras $T(V), T_{c}(W)$. The following is easy to see.@

Lemma 4.3. The canonical pairings $\langle\rangle:, T^{n}(V) \times T^{n}(W) \rightarrow \mathbb{k}, n \in \mathbb{N}$ defined by

$$
\left\langle v_{1} \otimes \cdots \otimes v_{n}, w_{1} \otimes \cdots \otimes w_{n}\right\rangle=\left\langle v_{1}, w_{1}\right\rangle \ldots\left\langle v_{n}, w_{n}\right\rangle
$$

give rise to an $\mathbb{N}$-homogeneous Hopf pairing $\langle\rangle:, T(V) \times T_{c}(W) \rightarrow \mathbb{k}$, which is obviously non-degenerate.

Let $C$ be an affine Hopf algebra, that is, a finitely generated commutative Hopf algebra. Set $J=C^{\circ}$, the dual cocommutative Hopf algebra of $C$; see Section 2.4. Let $\langle\rangle:, J \otimes C \rightarrow \mathbb{k}$ be the canonical pairing. Since $C$ is proper in the sense that the canonical map $C \rightarrow J^{*}$ is an injection (see [19, Theorem 6.1.3]), we have the following.

Lemma 4.4. $\langle\rangle:, J \otimes C \rightarrow \mathbb{k}$ is a non-degenerate Hopf pairing. 
Suppose that $W$ is a right $C$-comodule. Then $V$ has the transposed left $C$ comodule structure, which induces the right $J$-module structure determined by

$$
\langle v \triangleleft a, w\rangle=\sum\left\langle v, w_{(0)}\right\rangle\left\langle a, w_{(1)}\right\rangle, \quad v \in V, a \in J, w \in W .
$$

We have now the two $\mathbb{N}$-graded Hopf superalgebras $\mathcal{H}(J, V), \mathcal{A}(C, W)$. The following is verified directly.@

Proposition 4.5. The pairing $\langle\rangle:, \mathcal{H}(J, V) \times \mathcal{A}(C, W) \rightarrow \mathbb{k}$ defined by

$$
\langle a \otimes x, c \otimes z\rangle=\langle a, c\rangle\langle x, z\rangle, \quad a \in J, x \in T(V), c \in J, z \in T_{c}(W)
$$

is a non-degenerate $\mathbb{N}$-homogeneous Hopf pairing.

As was seen in (2.8), the pairing above naturally extends to

$$
\langle,\rangle: \mathcal{H}(J, V) \times \widehat{\mathcal{A}}(C, W) \rightarrow \mathbb{k} .
$$

One sees that $C$ is naturally a left $J$-module under the action $a \rightarrow c$, where $a \in J, c \in C$, defined by

$$
\langle b, a \rightarrow c\rangle=\langle b a, c\rangle, \quad b \in J .
$$

In the following, Hom $J$ represents the vector space of left $J$-linear maps, while Hom represents that of $\mathbb{k}$-linear maps. Note

$$
\operatorname{Hom}_{J}(\mathcal{H}(J, V), C)=\prod_{n=0}^{\infty} \operatorname{Hom}_{J}\left(J \otimes T^{n}(V), C\right) .
$$

Just as $\widehat{\mathcal{A}}(C, W)$, this can be regarded as the completion of the $\mathbb{N}$-graded vector space $\bigoplus_{n=0}^{\infty} \operatorname{Hom}_{J}\left(J \otimes T^{n}(V), C\right)$. Therefore we can construct the complete tensor product

$$
\begin{aligned}
& \operatorname{Hom}_{J}(\mathcal{H}(J, V), C) \widehat{\otimes} \operatorname{Hom}_{J}(\mathcal{H}(J, V), C) \\
& =\prod_{n, m \geq 0} \operatorname{Hom}_{J}\left(J \otimes T^{n}(V), C\right) \otimes \operatorname{Hom}_{J}\left(J \otimes T^{m}(V), C\right),
\end{aligned}
$$

which is naturally included in

$$
\begin{aligned}
& \operatorname{Hom}(\mathcal{H}(J, V) \otimes \mathcal{H}(J, V), C \otimes C) \\
& =\prod_{n, m \geq 0} \operatorname{Hom}\left(\left(J \otimes T^{n}(V)\right) \otimes\left(J \otimes T^{m}(V)\right), C \otimes C\right) .
\end{aligned}
$$

Proposition 4.6. (1) The linear isomorphisms $C \otimes T^{n}(W) \stackrel{\simeq}{\longrightarrow} \operatorname{Hom}_{J}(J \otimes$ $\left.T^{n}(V), C\right), n \geq 0$ given by

$$
c \otimes z \mapsto(a \otimes x \mapsto\langle x, z\rangle a \rightarrow c),
$$

amount to a linear homeomorphism,

$$
\xi: \widehat{\mathcal{A}}(C, W) \stackrel{\simeq}{\longrightarrow} \operatorname{Hom}_{J}(\mathcal{H}(J, V), C) .
$$

(2) Let $f, g \in \operatorname{Hom}_{J}(\mathcal{H}(J, V), C), X \in \mathcal{H}(J, V)$. The product, the unit, the counit $\widehat{\varepsilon}$ and the antipode $\widehat{S}$ on $\widehat{\mathcal{A}}(C, W)$ are transferred onto $\operatorname{Hom}_{J}(\mathcal{H}(J, V), C)$ 
via the $\xi$ above so that

$$
\begin{aligned}
f g(X) & =\sum f\left(X_{(1)}\right) g\left(X_{(2)}\right), \\
\xi(1)(X) & =\varepsilon(X) 1, \\
\widehat{\varepsilon}(f) & =\varepsilon(f(1)), \\
\langle a, \widehat{S}(f)(X)\rangle & =\sum\left\langle S\left(a_{(1)}\right), f\left(a_{(2)} S(X) S\left(a_{(3)}\right)\right)\right\rangle, \quad a \in J .
\end{aligned}
$$

(3) Via $\xi$ and $\xi \widehat{\otimes} \xi$, the coproduct on $\widehat{\mathcal{A}}(C, W)$ is translated to

$$
\begin{aligned}
\widehat{\Delta}: \operatorname{Hom}_{J}(\mathcal{H}(J, V), C) \rightarrow & \operatorname{Hom}_{J}(\mathcal{H}(J, V), C) \widehat{\otimes} \operatorname{Hom}_{J}(\mathcal{H}(J, V), C) \\
& \subset \operatorname{Hom}(\mathcal{H}(J, V) \otimes \mathcal{H}(J, V), C \otimes C)
\end{aligned}
$$

that is determined by

$$
\langle a \otimes b, \widehat{\Delta}(f)(X \otimes Y)\rangle=\sum\left\langle a b_{(1)}, f\left(S\left(b_{(2)}\right) X b_{(3)} Y\right)\right\rangle,
$$

where $a, b \in J, f \in \operatorname{Hom}_{J}(\mathcal{H}(J, V), C), X, Y \in \mathcal{H}(J, V)$.

Proof. (1) This is easy to see.

(2) We verify the formula for $\widehat{S}$; the rest is verified similarly. We may suppose that $f$, restricted to $J \otimes T^{n}(V)$, is given by

$$
f(b \otimes x)=\langle x, z\rangle b \rightarrow c, \quad b \in J, x \in T^{n}(V)
$$

for some $c \in C, z \in T^{n}(W)$. Recall from Proposition 4.2 the description of the antipode of $\mathcal{A}(C, W)$. Then one sees

$$
\widehat{S}(f)(b \otimes x)=\sum\left\langle x, S\left(z_{(0)}\right)\right\rangle b \rightarrow S\left(c z_{(1)}\right) .
$$

Therefore, the desired formula will follow if we see that for every $a \in J$,

$$
\begin{aligned}
& \sum\left\langle a b, S\left(c z_{(1)}\right)\right\rangle\left\langle x, S\left(z_{(0)}\right)\right\rangle \\
& =\sum\left\langle S\left(a_{(1)}\right), a_{(2)} S\left(b_{(1)}\right) S\left(a_{(3)}\right) \rightarrow c\right\rangle\left\langle S(x) \triangleleft S\left(b_{(2)}\right) S\left(a_{(4)}\right), z\right\rangle .
\end{aligned}
$$

Here one should recall from Proposition 3.8 the description of the antipode of $\mathcal{H}(J, V)$. Using the antipodal identity for $J$ and Equation (4.4), we compute that the right-hand side equals

$$
\sum\left\langle S\left(b_{(1)}\right) S\left(a_{(1)}\right), c\right\rangle\left\langle S(x), z_{(0)}\right\rangle\left\langle S\left(b_{(2)}\right) S\left(a_{(2)}\right), z_{(1)}\right\rangle,
$$

which is seen to equal the left-hand side by Lemmas 4.3, 4.4,

(3) We may suppose that $f$ is as above. Then the desired formula will follow if we see that

$$
\begin{aligned}
& \sum\left\langle a, g \rightarrow c_{(1)}\right\rangle\left\langle x,\left(z_{(1)}\right)_{(0)}\right\rangle\left\langle b, h \rightarrow\left(z_{(1)}\right)_{(1)} c_{(2)}\right\rangle\left\langle y, z_{(2)}\right\rangle \\
& =\sum\left\langle a b_{(1)}, S\left(b_{(2)}\right) g b_{(3)} h_{(1)} \rightarrow c\right\rangle\left\langle\left(x \triangleleft b_{(4)} h_{(2)}\right) y, z\right\rangle,
\end{aligned}
$$

where $a, b \in J$ and $g \otimes x, h \otimes y \in \mathcal{H}(J, V)$. We compute that the left-hand side equals

$$
\sum\left\langle a g, c_{(1)}\right\rangle\left\langle x \triangleleft b_{(1)} h_{(1)}, z_{(1)}\right\rangle\left\langle b_{(2)} h_{(2)}, c_{(2)}\right\rangle\left\langle y, z_{(2)}\right\rangle,
$$

which is seen to equal the right-hand side since $J$ is cocommutative. 
4.3. Let $C, J$ be as in the preceding subsection. Thus, $C$ is an affine Hopf algebra, and $J=C^{\circ}$. Set $G=\operatorname{Sp} C$, the algebraic affine group scheme represented by $C$. By definition the Lie algebra $P(J)$ of primitives in $J$ is the Lie algebra Lie $G$ of $G$. Recall $C^{+}=\operatorname{Ker} \varepsilon$. The quotient vector space $C^{+} /\left(C^{+}\right)^{2}$ of $C^{+}$divided by its square is the cotangent space of $G$ at 1 , and is dual to $P(J)$.

Let $J^{1}$ denote the irreducible component of $J$ containing 1; see Section 2.5. This consists of those elements in $C^{*}$ which annihilate some power $\left(C^{+}\right)^{n}$ of $C^{+}$. This is called the hyperalgebra of $G$, denoted hy $G=J^{1}$. One sees $P(J) \subset J^{1}$. Summarizing we have

$$
P\left(J^{1}\right)=P(J)=\operatorname{Lie} G=\left(C^{+} /\left(C^{+}\right)^{2}\right)^{*} .
$$

Remark 4.7. Consider the right adjoint coaction

$$
c \mapsto \sum c_{(2)} \otimes S\left(c_{(1)}\right) c_{(3)}, C \rightarrow C \otimes C
$$

by $C$ on itself; this corresponds to the right adjoint action by $G$ on itself. This induces on $C^{+} /\left(C^{+}\right)^{2}$ a right $C$-comodule structure, which in turn is transposed to a left $C$-comodule structure on $P(J)$. This last induces on $P(J)$ structures of right modules over $J, J^{1}$ and $G$. One sees that they coincide with the familiar adjoint actions.

If $G$ is connected, or equivalently if the prime spectrum $\operatorname{Spec} C$ of $C$ is connected, we say that $C$ is connected. The following is well-known; see [21, Proposition 0.3.1(g)].

Lemma 4.8. Suppose that $C$ is connected. Then the canonical pairing $\langle$,$\rangle :$ $J^{1} \times C \rightarrow \mathbb{k}$, which is restricted from $\langle\rangle:, J \times C \rightarrow \mathbb{k}$, is non-degenerate.

Remark 4.9. (1) If char $\mathbb{k}=0$, then $J^{1}=U(\operatorname{Lie} G)$; see Remark 3.2(2).

(2) Assume that $\mathbb{k}$ is algebraically closed. Then by Kostant's Theorem [19, Theorems 8.1.5], $J=\mathbb{k} G(\mathbb{k}) \ltimes J^{1}$, the smash (or semi-direct) product of $J^{1}$ by the group algebra of the linear algebraic group $G(\mathbb{k})$.

Remark 4.10. Suppose that we are in the situation of Proposition 4.6. We have the dual Harish-Chandra pair $\left(J^{1}, V\right)$ with the restricted action on $V$ by $J^{1}$, which gives rise to the Hopf superalgebra $\mathcal{H}\left(J^{1}, V\right)$; it is a Hopf super-subalgebra of $\mathcal{H}(J, V)$ such that $\mathcal{H}(J, V)=J \otimes_{J^{1}} \mathcal{H}\left(J^{1}, V\right)$ as a left $J$-module. This last implies

$$
\operatorname{Hom}_{J}(\mathcal{H}(J, V), C)=\operatorname{Hom}_{J^{1}}\left(\mathcal{H}\left(J^{1}, V\right), C\right) .
$$

Therefore, in Proposition 4.5 we may replace the former with the latter. Moreover, in the formulas for $\widehat{S}, \widehat{\Delta}$, we may suppose by Lemma 4.8 that $a, b \in J^{1}$, if $G$ is connected. We may suppose that $a, b \in G(\mathbb{k})$, if $\mathbb{k}$ is an algebraically closed field of characteristic zero, since $C$ is then the algebra of all polynomial functions on the linear algebraic group $G(\mathbb{k})$. In this last situation the resulting formulas are essentially the same as those given by Carmeli and Fioresi [8, Proposition 3.10]. But, contrary to the method of [8] or [26], we will not depend on the formulas to construct affine Hopf superalgebras (or algebraic affine supergroup schemes) from Harish-Chandra pairs. Especially, we cannot use the formula (4.6) to define their coproducts. For we do not see that $\widehat{\Delta}(f)(X \otimes Y)$ has its value in $C \otimes C$, because we do 
not have such a characterization as in the situation of [8] that $C \otimes C$ is the algebra of the polynomial functions on $G(\mathbb{k}) \times G(\mathbb{k})$.

4.4. Let $C$ be an affine Hopf algebra, and let $W$ be a finite-dimensional right $C$-comodule. Set

$$
G=\operatorname{Sp} C, \quad J=C^{\circ}, \quad V=W^{*},
$$

as in the preceding two subsections. Recall from (4.4) that $V$ is naturally a right $J$-module.

Definition 4.11. $(C, W)$ is called a Harish-Chandra pair, if it is equipped, as its structure, with a bilinear map [ , ] $V \times V \rightarrow P(J)$ with which $(J, V)$ is a dual Harish-Chandra pair; see Definition 3.1. It is said to be connected, if $C$ is connected.

Suppose that $\left(C^{\prime}, W^{\prime}\right)$ is another Harish-Chandra pair with $J^{\prime}=\left(C^{\prime}\right)^{\circ}$, $V^{\prime}=\left(W^{\prime}\right)^{*}$. A morphism $(C, W) \rightarrow\left(C^{\prime}, W^{\prime}\right)$ of Harish-Chandra pairs is a pair of a Hopf algebra map $f: C \rightarrow C^{\prime}$ and a linear map $g: W \rightarrow W^{\prime}$ such that the dual Hopf algebra map $f^{\circ}: J^{\prime} \rightarrow J$ of $f$ and the dual linear map $g^{*}: V^{\prime} \rightarrow V$ constitute a morphism $\left(J^{\prime}, V^{\prime}\right) \rightarrow(J, V)$ of dual HarishChandra pairs.

The Harish-Chandra pairs and their morphisms naturally form a category $\mathrm{HCP}$. We let cHCP denote the full subcategory of HCP consisting of all connected Harish-Chandra pairs.

In the situation above, the object $(J, V)$ (resp., the morphism $\left.\left(f^{\circ}, g^{*}\right)\right)$ in DHCP is said to be associated with the object $(C, W)$ (resp., the morphism $(f, g))$ in $\mathrm{HCP}$.

Remark 4.12. (1) In the situation above, Condition (a) of Definition 3.1 is equivalent to the condition that [ , ], regarded as a linear map $V \otimes V \rightarrow$ $P(J)$, is left $C$-colinear; see Remark 4.7. One sees from Lemma 4.8 that if $C$ is connected, then the last condition is equivalent to that [, ] is left $J^{1}$-linear. It follows that if $C$ is connected, then $(C, W)$ is a Harish-Chandra pair if the associated bilinear map makes $\left(J^{1}, V\right)$ into a dual Harish-Chandra pair.

(2) Suppose char $\mathbb{k}=0$. We see from Proposition 3.3 and the first half of Part 1 above that HCP is anti-isomorphic to the category of those pairs $(G, L)$ of an algebraic affine group scheme $G$ and a finite-dimensional Lie superalgebra $L$, given a rational right $G$-module structure on $L_{1}$, which satisfy

(i) $L_{0}=\operatorname{Lie} G$,

(ii) the right adjoint action on $L_{1}$ by $L_{0}$ coincides with the action arising from the given rational right $G$-module structure, and

(iii) the bracket [, ] : $L_{1} \times L_{1} \rightarrow L_{0}$ on $L$ restricted to $L_{1}$ is $G$ equivariant, where $L_{0}$ is regarded as a rational right $G$-module by the adjoint action.

In [8] and others, the latter category is defined as the category of (super) Harish-Chandra pairs. More precisely, they assume in addition that $\mathbb{k}$ is algebraically closed, to identify rational $G$-modules with rational modules over the linear algebraic group $G(\mathbb{k})$. 
Proposition 4.13. Suppose char $\mathbb{k}=0$. Then $\mathrm{cHCP}$ is anti-isomorphic to the category of the pairs $(G, L)$ of a connected algebraic affine group scheme $G$ and a finite-dimensional Lie superalgebra $L$ such that $L_{0}=\operatorname{Lie} G$, and the adjoint action on $L_{1}$ by $L_{0}$ arises (necessarily uniquely) from a rational $G$-module structure.

Proof. This follows from Lemma 4.8 and the second half of Remark 4.12(1).

In general we have the following, which follows from definitions and Lemmas 4.4, 4.8.

Proposition 4.14. (1) $(C, W) \mapsto\left(C^{\circ}, W^{*}\right)$ defines a contravariant functor,

$$
-^{\circ}: \mathrm{HCP} \rightarrow \mathrm{DHCP} \text {, }
$$

which is faithful.

(2) $(C, W) \mapsto\left(\left(C^{\circ}\right)^{1}, W^{*}\right)$ defines a contravariant functor,

$$
\left(-^{\circ}\right)^{1}: \mathrm{HCP} \rightarrow \mathrm{iDHCP},
$$

which is faithful when it is restricted to $\mathrm{CHCP}$.

4.5. Suppose that $A$ is a super-commutative Hopf superalgebra. Let $I_{A}=$ $A A_{1}$ be the super-ideal of $A$ generated by the odd part $A_{1}$; this is the smallest super-ideal such that $A / I_{A}$ is an ordinary algebra. Recall $A^{+}=\operatorname{Ker} \varepsilon$, and let $A_{0}^{+}=A_{0} \cap A^{+}$. We define $\bar{A}, W^{A}$, as in [15], by

$$
\bar{A}:=A / I_{A}=A_{0} / A_{1}^{2}, \quad W^{A}:=\left(A^{+} /\left(A^{+}\right)^{2}\right)_{1}=A_{1} / A_{0}^{+} A_{1} .
$$

One sees that $\bar{A}$ is indeed an ordinary quotient Hopf algebra of $A$. Let $G=\operatorname{SSp} A$. Then $\bar{A}$ represents an affine group scheme,

$$
G_{\mathrm{res}}=\operatorname{Sp} \bar{A},
$$

which is the group-valued functor obtained from $G$ by restricting its domain to the category of commutative algebras; see [17]. We call $G_{\text {res }}$ the affine group scheme associated with $G$.

One sees that $W^{A}$ is the odd part of the cotangent space $A^{+} /\left(A^{+}\right)^{2}$ of $G$ at 1. It is known that $A$ is finitely generated if and only if $\bar{A}$ is finitely generated, $\operatorname{dim} W^{A}<\infty$ and the ideal $I_{A}$ of $A$ is nilpotent; see [15, Proposition 4.4]. One sees from [15, Theorem 4.5] (see also Proposition 4.15 below) that $A$ or $G$ is purely even if and only if $W^{A}=0$.

Let $A \in \mathrm{AHSA}$, and set $H=A^{\circ}$. Then, $H \in \mathrm{CCSHA}$. We see that the canonical pairing $\langle\rangle:, H \times A \rightarrow \mathbb{k}$ is a Hopf pairing. Since $\left\langle\underline{H}, A_{1}\right\rangle=0$, a Hopf pairing $\underline{H} \times \bar{A} \rightarrow \mathbb{k}$ is induced.

Recall from Section 3.1 the definitions of $\underline{H}, V_{H}$. One sees from [15, Proposition 4.3]

$$
V_{H}=\left(W^{A}\right)^{*} .
$$

Therefore, we have the canonical Hopf pairing $\langle\rangle:, \wedge\left(V_{H}\right) \times \wedge\left(W^{A}\right) \rightarrow \mathbb{k}$, as given by (2.5).

Choose a totally ordered basis $X$ of $V_{H}$. Recall from Proposition 3.12 that we have the isomorphism $\phi_{X}: \underline{H} \otimes \wedge\left(V_{H}\right) \stackrel{\simeq}{\longrightarrow} H$. 
Proposition 4.15. There exists uniquely a counit-preserving, left $\bar{A}$-colinear isomorphism $\psi_{X}: A \stackrel{\simeq}{\longrightarrow} \bar{A} \otimes \wedge\left(W^{A}\right)$ of superalgebras, such that

$$
\left\langle\phi_{X}(h \otimes u), a\right\rangle=\left\langle h \otimes u, \psi_{X}(a)\right\rangle, \quad h \in \underline{H}, u \in \wedge\left(V_{H}\right), a \in A .
$$

Proof. Define a unit-preserving super-coalgebra map $\iota_{X}: \wedge\left(V_{H}\right) \rightarrow H$ by

$$
\iota_{X}\left(x_{1} \wedge \cdots \wedge x_{n}\right)=x_{1} \ldots x_{n}
$$

where $x_{i} \in X, x_{1}<\cdots<x_{n}, 0 \leq n \leq \# X$. Then we have uniquely a counit-preserving superalgebra map $\pi_{X}: A \rightarrow \wedge\left(W^{A}\right)$ that satisfies

$$
\left\langle\iota_{X}(u), a\right\rangle=\left\langle u, \pi_{X}(a)\right\rangle, \quad u \in \wedge\left(V_{H}\right), a \in A .
$$

Define $\psi_{X}: A \rightarrow \bar{A} \otimes \wedge\left(W^{A}\right)$ by

$$
\psi_{X}(a)=\sum \bar{a}_{(1)} \otimes \pi_{X}\left(a_{(2)}\right) .
$$

Here and in what follows, $a \mapsto \bar{a}$ denotes the quotient map $A \rightarrow \bar{A}$. It is shown in the proof of [15, Theorem 4.5] that $\psi_{X}$ is an isomorphism. One sees easily that it has the desired properties.

Corollary 4.16. We have $\underline{H}=\bar{A}^{\circ}$, and the canonical pairing $\langle\rangle:, H \times$ $A \rightarrow \mathbb{k}$ is non-degenerate.

Proof. This corollary follows immediately from Proposition 4.15,

Lemma 4.17. Keep the situation as above. The right adjoint $\bar{A}$-coaction on $A$

$$
a \mapsto \sum a_{(2)} \otimes S\left(\bar{a}_{(1)}\right) \bar{a}_{(3)}, A \rightarrow A \otimes \bar{A}
$$

induces on $W^{A}$ a right $\bar{A}$-comodule structure.

Proof. This is seen since the structure map $A \rightarrow A \otimes \bar{A}$ is a superalgebra map which is compatible with the counit $\varepsilon: A \rightarrow \mathbb{k}$.

The induced coaction as well will be called the adjoint coaction. We will regard $W^{A}$ as a right $\bar{A}$-comodule by this coaction. It is transposed to a left $\bar{A}$-comodule structure on $V_{H}=\left(W^{A}\right)^{*}$.

Keep the situation as above. We obtain $\left(\underline{H}, V_{H}\right) \in \mathrm{DHCP}$ from $H=A^{\circ}$; see Proposition 3.4. Similarly to Remark 4.7, we see that the right module structure by $\underline{H}=\bar{A}^{\circ}$ on $V_{H}$ which is induced from the transposed left $\bar{A}$ comodule structure coincides with the right adjoint $\underline{H}$-action. This proves the first half of the following.

Proposition 4.18. $\left(\bar{A}, W^{A}\right)$, given the bilinear map [, ] : $V_{H} \times V_{H} \rightarrow$ $P(\underline{H})$ associated with $\left(\underline{H}, V_{H}\right) \in \mathrm{DHCP}$, is a Harish-Chandra pair. This construction is functorial, so that $A \mapsto\left(\bar{A}, W^{A}\right)$ gives a functor AHSA $\rightarrow$ HCP.

Proof. A morphism $f: A \rightarrow B$ in AHSA induces naturally a Hopf algebra map $\bar{f}: \bar{A} \rightarrow \bar{B}$ and a linear map $W^{f}: W^{A} \rightarrow W^{B}$. It follows by the functoriality shown in [15, Remark 4.8] and in Proposition 3.4 that the two maps form a morphism in DHCP, since their duals $\bar{f}^{\circ},\left(W^{f}\right)^{*}$ coincide with the maps which arise from the morphism $f^{\circ}: B^{\circ} \rightarrow A^{\circ}$ in CCHSA. This implies the desired functoriality. 
Remark 4.19. For later use, recall from 15 that given $A \in$ AHSA, we have the $\mathbb{N}$-graded Hopf superalgebra

$$
\operatorname{gr} A:=\bigoplus_{n=0}^{\infty} I_{A}^{n} / I_{A}^{n+1} .
$$

The adjoint $\bar{A}$-coaction on $W^{A}$ extends uniquely to $\wedge\left(W^{A}\right) \rightarrow \wedge\left(W^{A}\right) \otimes \bar{A}$ with which $\wedge\left(W^{A}\right)$ is a Hopf-algebra object in $\operatorname{SMod}^{\bar{A}}$; see Lemma 4.1. Just as in (4.2), the smash coproduct constructs an $\mathbb{N}$-graded Hopf superalgebra, $\bar{A} \ltimes \wedge\left(W^{A}\right)$, with the algebra structure of tensor product. By [15, Proposition 4.9(2)], there exists uniquely a natural isomorphism $\psi: \operatorname{gr} A \stackrel{\simeq}{\longrightarrow}$ $\bar{A} \ltimes \wedge\left(W^{A}\right)$ such that $\psi(0): \operatorname{gr} A(0)=\bar{A} \rightarrow \bar{A}$ is the identity on $\bar{A}$, and $\psi(1): \operatorname{gr} A(1)=A_{1} / A_{1}^{3} \rightarrow \bar{A} \otimes W^{A}$ induces, with $A_{0} / A_{0}^{+} \otimes_{A_{0}}$ applied, the identity on $W^{A}$; cf. Proposition 4.15.

4.6. We wish to construct a quasi-inverse of the functor given by Proposition 4.18. Let $(C, W) \in \mathrm{HCP}$, and set $J=C^{\circ}, V=W^{*}$. Then $(J, V) \in$ $\mathrm{DHCP}$. Recall $\mathcal{H}(J, V), \widehat{\mathcal{A}}(C, W)$ and their pairing $\langle\rangle:, \mathcal{H}(J, V) \times \widehat{\mathcal{A}}(C, W) \rightarrow$ $\mathbb{k}$; see (3.3), (4.3) and (4.5). Recall also the Hopf super-ideal $I(J, V)$ of $\mathcal{H}(J, V)$ which defines $H(J, V)$ by $H(J, V)=\mathcal{H}(J, V) / I(J, V)$; see (3.4). We define

$$
A(C, W):=\{Z \in \widehat{\mathcal{A}}(C, W) \mid\langle I(J, V), Z\rangle=0\} .
$$

Lemma 4.20. Keep the notation as above.

(1) In the complete topological Hopf superalgebra $\widehat{\mathcal{A}}(C, W), A(C, W)$ is a super-subalgebra, and is stable under the antipode $\widehat{S}$.

(2) $A(C, W)$ is discrete in $\widehat{\mathcal{A}}(C, W)$, so that we have

$$
A(C, W) \otimes A(C, W)=A(C, W) \widehat{\otimes} A(C, W) \subset \widehat{\mathcal{A}}(C, W) \widehat{\otimes} \widehat{\mathcal{A}}(C, W) .
$$

Moreover, the coproduct $\widehat{\Delta}$ on $\widehat{\mathcal{A}}(C, W)$ induces $A(C, W) \rightarrow A(C, W) \otimes$ $A(C, W)$.

(3) Given the structure induced from $\widehat{\mathcal{A}}(C, W)$ as above, $A(C, W)$ is a super-commutative Hopf superalgebra.

Proof. (1) This follows since $I(J, V)$ is a super-coideal, and is stable under the antipode.

(2) Since $H(J, V)$ is finitely generated as a left $J$-module, it follows that if we set $M=\bigoplus_{i<n} J \otimes T^{i}(V)$ for $n$ large enough, then $\mathcal{H}(J, V)=M+I(J, V)$. If follows that $A(C, W)$ trivially intersects with $\prod_{i>n} C \otimes T^{n}(W)$; this shows that $A(C, W)$ is discrete since this product is an open neighborhood of 0 .

For simplicity let us write $I, \mathcal{H}, \widehat{\mathcal{A}}$ for $I(J, V), \mathcal{H}(J, V), \widehat{\mathcal{A}}(C, W)$. Consider the pairing on $\mathcal{H} \times \widehat{\mathcal{A}}$ and the induced one on $\mathcal{H} \otimes \mathcal{H} \times \widehat{\mathcal{A}} \widehat{\otimes} \widehat{\mathcal{A}}$. Given a subset $F$ in $\mathcal{H}$ or in $\mathcal{H} \otimes \mathcal{H}$, let $F^{\perp}$ denote the subset of $\widehat{\mathcal{A}}$ or of $\widehat{\mathcal{A}} \widehat{\otimes} \widehat{\mathcal{A}}$ consisting of those elements which annihilate $F$ with respect to the relevant pairing. Then $I^{\perp}=A(C, W)$. We see that in $\widehat{\mathcal{A}} \widehat{\otimes} \widehat{\mathcal{A}}$,

$$
\begin{aligned}
& (\mathcal{H} \otimes I+I \otimes \mathcal{H})^{\perp}=(\mathcal{H} \otimes I)^{\perp} \cap(I \otimes \mathcal{H})^{\perp} \\
& =\widehat{\mathcal{A}} \widehat{\otimes} I^{\perp} \cap I^{\perp} \widehat{\otimes} \widehat{\mathcal{A}}=I^{\perp} \widehat{\otimes} I^{\perp}=I^{\perp} \otimes I^{\perp} .
\end{aligned}
$$


Here the last equality holds true since $I^{\perp}=A(C, W)$ is discrete, as was shown above. The desired result follows since $I$ is an ideal, and so $\widehat{\Delta}\left(I^{\perp}\right) \subset$ $(\mathcal{H} \otimes I+I \otimes \mathcal{H})^{\perp}$.

(3) This follows easily from the results just proven.

Keep the notation as above. For the following two results, set

$$
A=A(C, W), \quad \mathcal{A}=\mathcal{A}(C, W), \quad \widehat{\mathcal{A}}=\widehat{\mathcal{A}}(C, W) .
$$

Choose a totally ordered basis $X$ of $V$, and define a unit-preserving supercoalgebra map $\widetilde{\iota}_{X}: \wedge(V) \rightarrow T(V)$, and a counit-preserving superalgebra map $\tilde{\pi}_{X}: T_{c}(W) \rightarrow \wedge(W)$, analogous to $\iota_{X}, \pi_{X}$ (see (4.10), (4.11)), as follows:

$$
\begin{aligned}
\widetilde{\iota}_{X}\left(x_{1} \wedge \cdots \wedge x_{n}\right\rangle & =x_{1} \otimes \cdots \otimes x_{n}, \\
\left\langle\widetilde{\iota}_{X}(u), Z\right\rangle & =\left\langle u, \widetilde{\pi}_{X}(Z)\right\rangle,
\end{aligned}
$$

where $x_{i} \in X, x_{1}<\cdots<x_{n}, 0 \leq n \leq \# X, u \in \wedge(V), Z \in T_{c}(W)$. Vanishing on $\prod_{n>\# X} T^{n}(W), \widetilde{\pi}_{X}$ is continuous, where $\wedge(W)$ is regarded as to be discrete, and hence it gives rise to the complete tensor product $\operatorname{id}_{C} \widehat{\otimes} \widetilde{\pi}_{X}: C \widehat{\otimes} T_{c}(W) \rightarrow C \otimes \wedge(W)$ of continuous linear maps.

Lemma 4.21. (1) Let

$$
\psi_{X}^{\prime}: A \hookrightarrow \widehat{\mathcal{A}}=C \widehat{\otimes} T_{c}(W) \rightarrow C \otimes \wedge(W)
$$

be the composite of the inclusion with $\operatorname{id}_{C} \widehat{\otimes} \widetilde{\pi}_{X}$. Then this is a counitpreserving isomorphism of superalgebras, such that

$$
\left\langle h \otimes \widetilde{\iota}_{X}(u), a\right\rangle=\left\langle h \otimes x, \psi_{X}^{\prime}(a)\right\rangle, \quad h \in J, u \in \wedge(V), a \in A
$$

(2) We have $A=A(C, W) \in \mathrm{AHSA}$.

Proof. (1) Recall from Proposition 4.6 the isomorphism $\xi: \widehat{\mathcal{A}}(C, W) \stackrel{\simeq}{\longrightarrow}$ $\operatorname{Hom}_{J}(\mathcal{H}(J, V), C)$. By using Lemma 4.4, one sees that this $\xi$ induces an isomorphism $A(C, W) \stackrel{\simeq}{\longrightarrow} \operatorname{Hom}_{J}(H(J, V), C)$; this is a key ingredient borrowed from Koszul [13]. Via these isomorphisms together with the one $\operatorname{Hom}_{J}(J \otimes \wedge(V), C) \stackrel{\longrightarrow}{\longrightarrow} C \otimes \wedge(W)$ that arises from the canonical pairing (see (2.5) ), the map $\psi_{X}^{\prime}$ is identified with $\operatorname{Hom}_{J}\left(\phi_{X}^{\prime}, C\right)$, where $\phi_{X}^{\prime}$ is the composite $J \otimes \wedge(V) \rightarrow J \otimes T(V)=\mathcal{H}(J, V) \rightarrow H(J, V)$ of $\operatorname{id}_{J} \otimes \widetilde{\iota}_{X}$ with the quotient map. Since $\phi_{X}^{\prime}$ is an isomorphism by Lemma 3.11, the desired result follows.

(2) This follows since the isomorphism just obtained shows that $A$ is finitely generated.

From Remark 4.19 and (2.7), recall the construction of

$\operatorname{gr} A=\bigoplus_{n \geq 0} I_{A}^{n} / I_{A}^{n+1} \simeq \bar{A} \ltimes \wedge\left(W^{A}\right), \quad \operatorname{gr} \widehat{\mathcal{A}}=\bigoplus_{n=0}^{\infty} \widehat{\mathcal{I}}_{n} / \widehat{\mathcal{I}}_{n+1}=\mathcal{A}=C \ltimes T_{c}(W)$,

where we set $\widehat{\mathcal{I}}_{n}=\prod_{i>n} C \otimes T^{i}(W)$ in $\widehat{\mathcal{A}}$. One sees that $I_{A} \subset \widehat{\mathcal{I}}_{1}$, whence $I_{A}^{n} \subset \widehat{\mathcal{I}}_{1}^{n} \subset \widehat{\mathcal{I}}_{n}$ for every $n>0$. Therefore the inclusion $A \hookrightarrow \widehat{\mathcal{A}}$ induces, with gr applied, a Hopf superalgebra map gr $A \rightarrow \mathcal{A}$ which preserves the $\mathbb{N}$-grading. 
Proposition 4.22. (1) The $\mathbb{N}$-graded map just obtained is isomorphic in degrees 0 , 1 , so that we have a Hopf algebra isomorphism $\bar{A} \stackrel{\simeq}{\longrightarrow} C$, and a $C$-colinear isomorphism $W^{A} \stackrel{\simeq}{\longrightarrow} W$, where the right $\bar{A}$-comodule $W^{A}$ is regarded as a right $C$-comodule along the first isomorphism.

(2) The canonical pairing on $\mathcal{H}(J, V) \times \widehat{\mathcal{A}}(C, W)$ given in (4.5) induces a non-degenerate Hopf pairing

$$
\langle,\rangle: H(J, V) \times A(C, W) \rightarrow \mathbb{k},
$$

which induces an isomorphism $H(J, V) \stackrel{\simeq}{\longrightarrow} A(C, W)^{\circ}$ of Hopf superalgebras. The Hopf pairing and the induced isomorphism are both natural in $(C, W)$.

(3) The pair of the isomorphisms obtained in Part 1 gives a natural isomorphism $\left(\bar{A}, W^{A}\right) \stackrel{\simeq}{\longrightarrow}(C, W)$ in $\mathrm{HCP}$.

Proof. (1) Apply to the trivial gr construction to $C \otimes \wedge(W)$ with respect to the descending chain $\bigoplus_{i \geq n} C \otimes \wedge^{i}(W), n=0,1, \ldots$, of super-ideals; these super-ideals are the powers of the kernel of the projection $C \otimes \wedge(W) \rightarrow$ $C$. The resulting $\operatorname{gr}(C \otimes \wedge(W))$ coincides with the original $C \otimes \wedge(W)$. Commuting with the projection onto $C, \operatorname{id}_{C} \widehat{\otimes} \widetilde{\pi}_{X}$ induces an $\mathbb{N}$-graded algebra map, gr $\widehat{\mathcal{A}}=\mathcal{A} \rightarrow \operatorname{gr}(C \otimes \wedge(W))=C \otimes \wedge(W)$, which is seen to be isomorphic in degrees 0,1 . Since gr $\psi_{X}^{\prime}$ is an isomorphism by Lemma 4.21 (1), the desired result follows.

(2) Since the tensor product of the canonical pairings on $J \times C, \wedge(V) \times$ $\wedge(W)$ is non-degenerate, and induces an isomorphism $J \otimes \wedge(V) \stackrel{\simeq}{\longrightarrow}(C \otimes$ $\wedge(W))^{\circ}$, the desired result follows from Lemma $4.21(1)$.

(3) Since the two isomorphisms both commute with the natural projections from $A(C, W)$, their duals $J=C^{\circ} \stackrel{\simeq}{\longrightarrow} \bar{A}^{\circ}, V=W^{*} \stackrel{\simeq}{\longrightarrow}\left(W^{A}\right)^{*}$ both extend to the isomorphism $H(J, V) \stackrel{\simeq}{\longrightarrow} A(C, W)^{\circ}$ obtained in Part 2. This shows that they give an isomorphism in HCP. The naturality is easy to see.

Theorem 4.23. The assignment $(C, W) \mapsto A(C, W)$ is functorial, so that we have the functor

$$
A: \mathrm{HCP} \rightarrow \mathrm{AHSA},(C, W) \mapsto A(C, W) .
$$

This is an equivalence which is a quasi-inverse of the functor $A \mapsto\left(\bar{A}, W^{A}\right)$ given by Proposition 4.18.

Proof. The functoriality is easy to see. Proposition $4.22(3)$ shows that the functor $A$ followed by $A \mapsto\left(\bar{A}, W^{A}\right)$ is isomorphic to the identity functor on HCP.

To complete the proof let $A \in \mathrm{AHSA}$, and set $(C, W):=\left(\bar{A}, W^{A}\right)$ in HCP. Let $\varpi: A \rightarrow A_{1} \rightarrow A_{1} / A_{0}^{+} A_{1}=W$ be the composite of the canonical projections. For each $n>0$, define $\varpi^{(n)}: A \rightarrow T^{n}(W)$ by

$$
\varpi^{(n)}(a)=\sum \varpi\left(a_{(1)}\right) \otimes \varpi\left(a_{(2)}\right) \otimes \cdots \otimes \varpi\left(a_{(n)}\right) .
$$

For $n=0$, let $\varpi^{(0)}=\varepsilon$. Define a map by

$$
\beta_{A}: A \rightarrow \widehat{\mathcal{A}}(C, W)=C \widehat{\otimes} T_{c}(W), \beta_{A}(a)=\sum_{n=0}^{\infty} \sum \bar{a}_{(1)} \otimes \varpi^{(n)}\left(a_{(2)}\right) .
$$


This is natural in $A$. It suffices to prove that $\beta_{A}$ gives a Hopf superalgebra isomorphism from $A$ onto $A(C, W)$.

Suppose that $(J, V) \in \mathrm{DHCP}$ is the pair associated with $(C, W)$. Let $H=A^{\circ}$. By (the proof of) Proposition 4.18 we have $(J, V)=\left(\underline{H}, V_{H}\right)$. Let $\gamma: \mathcal{H}(J, V) \rightarrow H(J, V) \stackrel{\simeq}{\longrightarrow} H$ be the composite of the quotient map with the natural isomorphism $\alpha_{H}$ given in (3.5); this is a Hopf superalgebra map. One sees that

$$
\langle\gamma(h \otimes u), a\rangle=\left\langle h \otimes u, \beta_{A}(a)\right\rangle, \quad h \in J, u \in T(V), a \in A .
$$

It follows from Corollary 4.16 that $\beta_{A}$ is an injective Hopf superalgebra map into $A(C, W)$. The image of $\beta_{A}$ is precisely $A(C, W)$, as desired, since given a totally ordered basis $X$ of $V$, the composite $A \rightarrow A(C, W) \stackrel{\simeq}{\longrightarrow} C \otimes \wedge(W)$ of $\beta_{A}$ with $\psi_{X}^{\prime}$ coincides with the isomorphism $\psi_{X}$.

Definition 4.24. An affine Hopf superalgebra $A$ is said to be connected if $\bar{A}$, or equivalently the prime spectrum $\operatorname{Spec} A_{0}$ of $A_{0}$, is connected. An algebraic affine supergroup scheme $G=\mathrm{SSp} A$ is said to be connected, if $A$ is connected.

Obviously, the equivalence obtained above restricts to a category equivalence between $\mathrm{cHCP}$ and the full subcategory of AHSA consisting of all connected ones.

\section{Duality and short exact Sequences}

5.1. Let $G=\operatorname{SSp} A$ be an algebraic affine supergroup scheme. Set $H=A^{\circ}$. Then $H \in$ CCHSA. Moreover, the primitives $P(H)$ form a finite-dimensional Lie superalgebra, and the irreducible component $H^{1}$ containing 1 is a hypersuperalgebra; see Section 2.5. We write

$$
\text { Lie } G=P(H), \quad \text { hy } G=H^{1},
$$

and call these the Lie superalgebra, and the hyperalgebra of $G$, respectively. One sees that Lie, hy give functors, which will play an important role in the following section.

Proposition 5.1. Suppose that an algebraic affine supergroup scheme $G$ is represented by $A(C, W)$, where $(C, W) \in \mathrm{HCP}$. Let $(J, V)=\left(\left(C^{\circ}\right)^{1}, W^{*}\right)$ be the object in $\mathrm{iDHCP}$ which is associated, by the functor given in Proposition 4.14(2), with $(C, W)$.

(1) Lie $G$ is naturally isomorphic to the Lie superalgebra constructed on $P(J) \oplus V$ as in Remark 3.2(1).

(2) hy $G$ is naturally isomorphic to $H(J, V)$.

Proof. This follows immediately from the isomorphism given in Proposition 4.22(2). For Part 2 note that $H\left(C^{\circ}, V\right)^{1}=H(J, V)$.

5.2. Conversely, let us start with an object $(J, V) \in \mathrm{DHCP}$, where we assume $\operatorname{dim} V<\infty$. Construct $H(J, V) \in$ CCHSA.

Proposition 5.2. With the notation as above, set $A=H(J, V)^{\circ}$; this is a super-commutative Hopf superalgebra which is not necessarily finitely generated. 
(1) The restriction maps $A \rightarrow J^{\circ}, A \rightarrow V^{*}$ induce isomorphisms

$$
\bar{A} \stackrel{\simeq}{\longrightarrow} J^{\circ}, \quad W^{A} \stackrel{\simeq}{\longrightarrow} V^{*}
$$

of Hopf algebras, and of super-vector spaces, respectively.

(2) Choose arbitrarily a totally ordered basis $X$ of $V$, and let $\phi_{X}: J \otimes$ $\wedge(V) \stackrel{\simeq}{\longrightarrow} H(J, V)$ be the isomorphism as given by Proposition 3.12. Then we have uniquely a counit-preserving, left $\bar{A}$-colinear superalgebra isomorphism $\psi_{X}: A \stackrel{\simeq}{\longrightarrow} \bar{A} \otimes \wedge\left(W^{A}\right)$ that satisfies the same formula as (4.9), when we replace the pairings on $\underline{H} \times \bar{A}, V_{H} \times W^{A}$ in (4.9) with those on $J \times \bar{A}, V \times W^{A}$ which are obtained from the isomorphisms of Part 1 above.

Proof. Let $\phi_{X}$ be the isomorphism as in Part 2, and identify $H(J, V)$ with $J \otimes \wedge(V)$ via $\phi_{X}$. By [15, Proposition 3.9(2)], $H(J, V)$ has the natural filtration $F_{n}:=\bigoplus_{i \leq n} J \otimes \wedge^{i}(V), n=0,1, \ldots$, which is compatible with the structure so that in particular, $F_{n} F_{m} \subset F_{n+m}$ for all $n, m \geq 0$. It follows that if $I$ is a right ideal of $J$ (of cofinite dimension), then $I \otimes \wedge(V)$ is a right ideal of $H(J, V)$ (of cofinite dimension). Note that in general, the dual coalgebra $R^{\circ}$ of an algebra $R$ consists of those elements in $R^{*}$ which annihilate some cofinite-dimensional right ideal of $R$, since such a right ideal, say $\mathfrak{a}$, includes a cofinite-dimensional ideal; take the annihilators $\operatorname{Ann}(R / \mathfrak{a})$, for example. Then we see that $H(J, V)^{\circ}=J^{\circ} \otimes(\wedge(V))^{*} \simeq J^{\circ} \otimes \wedge\left(V^{*}\right)$, which proves the proposition.

Remark 5.3. Let $(J, V) \in$ DHCP with $\operatorname{dim} V<\infty$, as above. Set $C:=J^{\circ}$, $W:=V^{*}$. Then the right $J$-module structure on $V$ gives rise naturally to a right $C$-comodule structure on $W$ (see (4.4)), from which we can construct, as before, a complete topological Hopf superalgebra, $\widehat{\mathcal{A}}(C, W)$, together with a pairing, $\langle\rangle:, \mathcal{H}(J, V) \times \widehat{\mathcal{A}}(C, W) \rightarrow \mathbb{k}$. If we define $A(C, W)$ by the same formula as (4.13), then it follows, as before, that $A(C, W)$ is a supercommutative Hopf superalgebra. Note that during the argument in the preceding section, what was essentially used from Lemma 4.4 was not the non-degeneracy of the pairing $\langle\rangle:, J \times C \rightarrow \mathbb{k}$, but rather only the injectivity of $C \rightarrow J^{*}$, and that this last injectivity holds true in the present situation. Then we see from the proof of Theorem 4.23 that $H(J, V)^{\circ}$ is naturally isomorphic to the $A(C, W)$ just defined.

5.3. Given a map $q: H \rightarrow H^{\prime}$ of Hopf superalgebras, we define

$$
\begin{aligned}
H^{\mathrm{co} q} & :=\left\{x \in H \mid \sum x_{(1)} \otimes q\left(x_{(2)}\right)=x \otimes q(1)\right\}, \\
\text { resp., }{ }^{\operatorname{co} q} H & :=\left\{x \in H \mid \sum q\left(x_{(1)}\right) \otimes x_{(2)}=q(1) \otimes x\right\} .
\end{aligned}
$$

This is the left (resp., right) coideal super-subalgebra of $H$ consisting of all right (resp., left) coinvariants along $q$. If $H$ is super-cocommutative, then

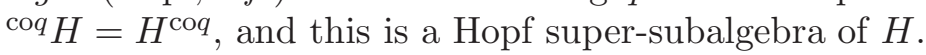

Let $H \in$ CCHSA. Recall that a Hopf super-subalgebra $K$ of $H$ is said to be normal [15, p.295], if $H K^{+}=K^{+} H$, or equivalently if $K$ is stable under the adjoint action, that is,

$$
\sum(-1)^{\left|x_{(1)}\right||y|} S\left(x_{(1)}\right) y x_{(2)} \in K, \quad x \in H, y \in K
$$


see [15. Theorem 3.10] for the equivalence of the conditions. If this is the case, $H / H K^{+}$is a quotient Hopf superalgebra of $H$. We write $H / / K$ for $H / H K^{+}$.

We say that an injective morphism $K \stackrel{p}{\rightarrow} H$ in CCHSA is normal if the image $p(K)$ of $K$ is normal in $H$.

Definition 5.4. A sequence $H_{1} \stackrel{p}{\rightarrow} H_{2} \stackrel{q}{\rightarrow} H_{3}$ in CCHSA is said to be short exact if the following equivalent conditions are satisfied:

(a) $p$ is an injection, and is normal, and $q$ induces an isomorphism $H_{2} / / p\left(H_{1}\right) \stackrel{\simeq}{\longrightarrow} H_{3}$

(b) $q$ is a surjection, and $p$ is an isomorphism $H_{1} \stackrel{\simeq}{\longrightarrow} H_{2}^{\operatorname{co} q}$ onto $H_{2}^{\operatorname{co} q}$. The equivalence above follows from [15, Theorem 3.10(3)].

Proposition 5.5. (1) The morphism $H(f, g): H\left(J_{1}, V_{1}\right) \rightarrow H\left(J_{2}, V_{2}\right)$ in CCHSA which arises from a morphism $(f, g):\left(J_{1}, V_{1}\right) \rightarrow\left(J_{2}, V_{2}\right)$ in DHCP is an injection (resp., a surjection) if and only if $f: J_{1} \rightarrow J_{2}$ and $g: V_{1} \rightarrow V_{2}$ are both injections (resp., surjections).

(2) The injective morphism $H(f, g): H\left(J_{1}, V_{1}\right) \rightarrow H\left(J_{2}, V_{2}\right)$ in CCHSA which arises from a morphism $(f, g):\left(J_{1}, V_{1}\right) \rightarrow\left(J_{2}, V_{2}\right)$ in DHCP, with $f$, $g$ injections, is normal if and only if (i) $f: J_{1} \rightarrow J_{2}$ is normal, (ii) $g\left(V_{1}\right)$ is $J_{2}$-stable in $V_{2}$, (iii) $\left[g\left(V_{1}\right), V_{2}\right] \subset f\left(J_{1}\right)$ and (iv) $v \triangleleft f(a)-\varepsilon(a) v \in g\left(V_{1}\right)$ for all $a \in J_{1}, v \in V_{2}$.

(3) The sequence $H\left(J_{1}, V_{1}\right) \rightarrow H\left(J_{2}, V_{2}\right) \rightarrow H\left(J_{3}, V_{3}\right)$ in CCHSA which arises from a sequence $\left(J_{1}, V_{1}\right) \rightarrow\left(J_{2}, V_{2}\right) \rightarrow\left(J_{3}, V_{3}\right)$ in DHCP is short exact if and only if the sequences $J_{1} \rightarrow J_{2} \rightarrow J_{3}, V_{1} \rightarrow V_{2} \rightarrow V_{3}$ which constitute the latter are short exact sequences of cocommutative Hopf algebras, and of vector spaces, respectively.

Proof. (1) This follows from Proposition 3.12, see also [15, Remark 3.8].

(2) This is seen from the construction of $H(J, V)$. Note that Condition (iv) is equivalent to that $f(a) v-v f(a) \in \operatorname{Im} H(f, g)$ for all $a \in J_{1}, v \in V_{2}$.

(3) This follows by Part 1 and [15, Theorem 3.13(3)].

5.4. Let $A$ be a super-commutative Hopf superalgebra. For every Hopf super-subalgebra $B \subset A, A B^{+}=B^{+} A$, and this is a Hopf super-ideal of $A$, so that we have a quotient Hopf superalgebra $A / A B^{+}$of $A$; we write $A / / B$ for $A / A B^{+}$.

Let $q: A \rightarrow B$ be a surjective morphism of super-commutative Hopf superalgebras. This is said to be conormal [15, Definition 5.7], if ${ }^{\operatorname{co} q} A=$ $A^{\mathrm{co} q}$, or equivalently if the kernel $\operatorname{Ker} q$ is $A$-costable, that is, sent to $\operatorname{Ker} q \otimes$ $A$, under the adjoint coaction $A \rightarrow A \otimes A$ given by

$$
a \mapsto \sum(-1)^{\left|a_{(1)}\right|\left|a_{(2)}\right|} a_{(2)} \otimes S\left(a_{(1)}\right) a_{(3)} ;
$$

see [15, Theorem 5.9] for the equivalence of the conditions. We have the closed embedding $N:=\operatorname{SSp} B \hookrightarrow G:=\operatorname{SSp} A$ of supergroup schemes which corresponds to $q$. One sees that $q$ is conormal if and only if for every supercommutative superalgebra $R$, the subgroup $N(R)$ of $G(R)$ is normal.

Definition 5.6. (1) A sequence $A_{1} \stackrel{p}{\rightarrow} A_{2} \stackrel{q}{\rightarrow} A_{3}$ of super-commutative Hopf superalgebras is said to be short exact if the following equivalent conditions are satisfied: 
(a) $p$ is an injection, and $q$ induces an isomorphism $A_{2} / / p\left(A_{1}\right) \stackrel{\simeq}{\longrightarrow} A_{3}$;

(b) $q$ is a surjection, and is conormal, and $p$ is an isomorphism $A_{1} \stackrel{\simeq}{\longrightarrow}$ $A_{2}^{\mathrm{co} q}$ onto $A_{2}^{\mathrm{co} q}$.

(2) A sequence $G_{1} \stackrel{\xi}{\rightarrow} G_{2} \stackrel{\eta}{\rightarrow} G_{3}$ of affine supergroup schemes is said to be short exact, if the following equivalent conditions are satisfied:

(a) $\eta$ is an epimorhism of dur sheaves, and $\xi$ gives an isomorphism $G_{1} \stackrel{\simeq}{\longrightarrow}$ Ker $\eta$

(b) $\xi$ is a closed embedding onto a closed normal super-subgroup, say $G_{1}^{\prime}$, of $G_{2}$, and $\eta$ induces an isomorphism $G_{2} \tilde{\tilde{\tilde{}}} G_{1}^{\prime} \stackrel{\simeq}{\longrightarrow} G_{3}$, where $G_{2} \tilde{\tilde{}} G_{1}^{\prime}$ denotes the dur sheafification of the 'naive' functor which associates to every super-commutative algebra $R$, the quotient group $G_{2}(R) / G_{1}^{\prime}(R)$; see [10, Chap. III, Sect. 3, 7.2], [28, Sect. 4].

This pair of equivalences, and the following proposition as well, follow from [15, Theorem 5.9] and [28, Proposition 4.2].

Proposition 5.7. Every short exact sequence $G_{1} \rightarrow G_{2} \rightarrow G_{3}$ of affine supergroup schemes arises uniquely from a short exact sequence $\mathcal{O}\left(G_{3}\right) \rightarrow$ $\mathcal{O}\left(G_{2}\right) \rightarrow \mathcal{O}\left(G_{1}\right)$ of super-commutative Hopf superalgebras, where $\mathcal{O}(G)$ denotes the super-commutative Hopf superalgebra which represents an affine supergroup scheme $G$.

Proposition 5.8. (1) The morphism $A(f, g): A\left(C_{1}, W_{1}\right) \rightarrow A\left(C_{2}, W_{2}\right)$ in AHSA which arises from a morphism $(f, g):\left(C_{1}, W_{1}\right) \rightarrow\left(C_{2}, W_{2}\right)$ in $\mathrm{HCP}$ is an injection (resp., a surjection) if and only if $f: C_{1} \rightarrow C_{2}$ and $g: W_{1} \rightarrow W_{2}$ are both injections (resp., surjections).

(2) Let $A(f, g): A\left(C_{2}, W_{2}\right) \rightarrow A\left(C_{3}, W_{3}\right)$ be a surjective morphism in AHSA which arises from a morphism $(f, g):\left(C_{2}, W_{2}\right) \rightarrow\left(C_{3}, W_{3}\right)$ in $\mathrm{HCP}$, with $f, g$ surjections. We know from Proposition 4.22(2) that the dual injective morphism $A(f, g)^{\circ}$ in CCHSA is naturally identified with $H\left(f^{\circ}, g^{*}\right)$ : $H\left(C_{3}^{\circ}, W_{3}^{*}\right) \rightarrow H\left(C_{2}^{\circ}, W_{2}^{*}\right)$ that arises from the morphism $\left(f^{\circ}, g^{*}\right)$ in DHCP associated with $(f, g)$. Then, $A(f, g)$ is conormal if and only if $f: C_{2} \rightarrow C_{3}$ is conormal, and $H\left(f^{\circ}, g^{*}\right)$ is normal.

(3) The sequence $A\left(C_{1}, W_{1}\right) \rightarrow A\left(C_{2}, W_{2}\right) \rightarrow A\left(C_{3}, W_{3}\right)$ in AHSA which arises from a sequence $\left(C_{1}, W_{1}\right) \rightarrow\left(C_{2}, W_{2}\right) \rightarrow\left(C_{3}, W_{3}\right)$ in $\mathrm{HCP}$ is short exact if and only if the sequences $C_{1} \rightarrow C_{2} \rightarrow C_{3}, W_{1} \rightarrow W_{2} \rightarrow W_{3}$ which constitute the latter are short exact sequences of commutative Hopf algebras, and of vector spaces, respectively.

Proof. (1) This follows from Proposition 4.15, see also [15, Remark 4.8].

Next, we prove first Part 3, and then Part 2.

(3) This follows by Part 1 and [15, Theorem 5.13(3)].

(2) If a closed super-subgroup $N \subset G$ of a affine super-group scheme $G$ is normal, then $N_{\text {res }}$ is normal in $G_{\text {res }}$; see (4.8). A conormal surjective morphism $q: A \rightarrow B$ of super-commutative Hopf superalgebras induces a normal injective morphism $q^{\circ}: B^{\circ} \rightarrow A^{\circ}$ of super-cocommutative Hopf superalgebras, since the condition that the adjoint $A$-coaction is induced onto $B$ along $q$ is dualized to that adjoint $A^{\circ}$-action on $A^{\circ}$ stabilizes $B^{\circ}$ 
through $q^{\circ}$. These together with Proposition 4.22(2) prove the 'only if' part.

To prove the 'if' part, assume the second condition, and set $C_{1}=C_{2}^{\operatorname{cof}}$, $W_{1}=\operatorname{Ker} g$. Note that $C_{1}$ is an affine Hopf algebra. Conditions (ii), (iv) of Proposition 5.5(2) imply that $W_{1}$ is a $C_{2}$-subcomodule of $W_{2}$, and is a right $C_{1}$-comodule. We regard $P\left(C_{2}^{\circ}\right) \supset P\left(C_{3}^{\circ}\right)$ via $f^{\circ}$. Condition (iii) implies that the structure [, ] : $W_{2}^{*} \times W_{2}^{*} \rightarrow P\left(C_{2}^{\circ}\right)$ of $\left(C_{2}, W_{2}\right)$ induces a bilinear map $W_{1}^{*} \times W_{1}^{*} \rightarrow P\left(C_{2}^{\circ}\right) / P\left(C_{3}^{\circ}\right)$. Since $0 \rightarrow P\left(C_{3}^{\circ}\right) \rightarrow P\left(C_{2}^{\circ}\right) \rightarrow$ $P\left(C_{1}^{\circ}\right)$ is exact, we can compose the induced bilinear map with a natural injection $P\left(C_{2}^{\circ}\right) / P\left(C_{3}^{\circ}\right) \hookrightarrow P\left(C_{1}^{\circ}\right)$ to obtain $W_{1}^{*} \times W_{1}^{*} \rightarrow P\left(C_{1}^{\circ}\right)$, which is $C_{1}$-colinear, as is easily seen. We see that $\left(C_{1}, W_{1}\right)$ together with the thus obtained bilinear map forms a Harish-Chandra pair, and is a subobject of $\left(C_{2}, W_{2}\right)$. By Part $3, A(f, g)$ extends to a short exact sequence $A\left(C_{1}, W_{1}\right) \rightarrow A\left(C_{2}, W_{2}\right) \rightarrow A\left(C_{3}, W_{3}\right)$, and hence is conormal.

\section{Simply CONNECTED AFFine SUPERGROUP SCHEMES}

Definition 6.1. Given a connected algebraic affine supergroup scheme $G$, an etale supergroup covering of $G$ is a pair $(F, \eta)$ of a connected algebraic affine supergroup scheme $F$ and an epimorphism $\eta: F \rightarrow G$ of supergroup sheaves such that the kernel Ker $\eta$ of $\eta$ is purely even, and is finite etale. A simply connected affine supergroup scheme is a connected algebraic affine supergroup scheme that has no non-trivial etale supergroup covering.

This directly generalizes the definition in the non-super situation; see 21, for example.

Proposition 6.2. A connected algebraic affine supergroup scheme $G$ is simply connected if and only if the associated affine group scheme $G_{\mathrm{res}}$ (see (4.8)), which is necessarily algebraic and connected, is simply connected.

Proof. Proposition 5.8(3), combined with Proposition 5.7, shows that if $E \rightarrow$ $F \rightarrow G$ is a short exact sequence of affine supergroup schemes with $E$ purely even, then it induces the short exact sequence $E \rightarrow F_{\text {res }} \rightarrow G_{\text {res }}$ of affine group schemes. This proves the 'if' part.

To prove the 'only if' part, suppose that we are given a short exact sequence of affine group schemes $E \rightarrow F_{0} \rightarrow G_{\text {res }}$ in which $E$ is finite etale, and $F_{0}$ is connected algebraic. It suffices to construct such a short exact sequence $E \rightarrow F \rightarrow G$ of affine supergroup schemes that induces the given short exact sequence. By [21, Proposition 1.1], $F_{0} \rightarrow G$ induces an isomorphism hy $F_{0} \stackrel{\simeq}{\longrightarrow}$ hy $G_{\text {res }}$. Suppose that $G$ corresponds to $(C, W) \in \mathrm{cHCP}$. Then we may suppose that the Hopf algebra $D:=\mathcal{O}\left(F_{0}\right)$ which represents $F_{0}$ includes $C$ as a Hopf subalgebra, so that $W$ may be regarded a right $D$-comodule. We see from the last isomorphism and Remark 4.12(1) that the structure [ , ] of $(C, W)$ makes $(D, W)$ into an object in cHCP including $(C, W)$ as a sub-object. If $F$ denotes the algebraic affine supergroup scheme corresponding to $(D, W)$, there arises a desired short exact sequence, again by Proposition $5.8(3)$.

Theorem 6.3. Suppose that $\mathbb{k}$ is an algebraically closed field of characteristic zero. Then $G \mapsto \operatorname{Lie} G$ gives rises to a bijection from the set of the 
isomorphism classes of all simply connected affine supergroup schemes $G$ to the set of the isomorphism classes of all those finite-dimensional Lie superalgebras $L$ such that (i) the radical $\operatorname{Rad} L_{0}$ of $L_{0}$ is nilpotent, and (ii) $\operatorname{ad}^{n}\left(\operatorname{Rad} L_{0}\right)\left(L_{1}\right)=0$ for some $n>0$.

Proof. In the non-super situation this was proved by Hochschild [11. According to [11, a connected algebraic affine group scheme $G=\operatorname{Sp} C$ with $L_{0}=$ Lie $G$ is simply connected if and only if (a) the radical of $L_{0}=P\left(C^{\circ}\right)$ is nilpotent, and (b) the canonical injective Hopf algebra map $C \rightarrow U\left(L_{0}\right)^{\circ}$ maps onto the Hopf subalgebra $B\left(L_{0}\right)$ of $U\left(L_{0}\right)^{\circ}$ consisting of those elements which annihilate some powers of the ideal $\left\langle\operatorname{Rad} L_{0}\right\rangle$ of $U\left(L_{0}\right)$ generated by the radical $\operatorname{Rad} L_{0}$. Moreover, $C \mapsto P\left(C^{\circ}\right)$ and $L_{0} \mapsto B\left(L_{0}\right)$ give a bijection, modulo isomorphism, between the set of all those connected affine Hopf algebras $C$ which satisfy the conditions (a), (b) and the set of all finitedimensional Lie algebras $L_{0}$ with nilpotent radical. Suppose that $C$ and $L_{0}$ correspond to each other. Then, given a finite-dimensional vector space $W$, all the right $C$-comodule structures on $W$ and all those right $U\left(L_{0}\right)$ module structures on $W^{*}$ such that $W^{*} \triangleleft\left\langle\operatorname{Rad} L_{0}\right\rangle^{n}=0$ for some $n>0$ are naturally in one-to-one correspondence. It follows by Remark 4.12(1) that $(C, W) \mapsto P\left(C^{\circ}\right) \oplus W^{*}$ gives a bijection, modulo isomorphism, from the set of all those object $(C, W) \in \mathrm{cHCP}$ such that $\mathrm{Sp} C$ is simply connected to the set of all those finite-dimensional Lie superalgebras $L$ which satisfy the conditions (i), (ii) above. This, combined with Theorem 4.23 and Proposition 6.2, proves the desired result.

Lemma 6.4. A super-cocommutative Hopf superalgebra $H$ such that $\operatorname{dim} V_{H}<$ $\infty$ is proper if and only if $\underline{H}$ is proper. Here, recall that an algebra $R$ is said to be proper if the canonical map $R \rightarrow\left(R^{\circ}\right)^{*}$ is an injection.

Proof. This is seen from Proposition 5.2 .

Definition 6.5. Generalizing directly the definition [21, p.258] in the nonsuper situation, we say that a hyper-superalgebra $H$ is of finite type, if the Lie superalgebra $P(H)$ is finite-dimensional. This is equivalent to the condition that the hyperalgebra $\underline{H}$ is of finite-type, and $\operatorname{dim} V_{H}<\infty$, since we have $P(H)=P(\underline{H}) \oplus V_{H}$.

Theorem 6.6. Suppose that $\mathbb{k}$ is a perfect field of positive characteristic $>2$. Then $G \mapsto$ hy $G$ gives an equivalence from the category of the simply connected affine supergroup schemes to the category of all those hypersuperalgebras $H$ of finite type such that (i) $\underline{H}^{\mathrm{ab}}$ is finite-dimensional, and (ii) $H$ is proper. Here, $\underline{H}^{\mathrm{ab}}$ is the abelianization of $\underline{H}$, that is, the quotient (indeed, Hopf) algebra of $\underline{H}$ divided by its commutator ideal.

Proof. In the non-super situation this was proved by Takeuchi 22, Corollary 5.4]. According to [22], a connected algebraic affine group scheme $G=\operatorname{Sp} C$ with $J=$ hy $G$ is simply connected if and only if the canonical Hopf algebra map $C \rightarrow J^{\circ}$ is an isomorphism. Moreover, $C \mapsto\left(C^{\circ}\right)^{1}$ and $J \mapsto J^{\circ}$ give an anti-equivalence between the category of all those connected affine Hopf algebras $C$ such that $\operatorname{Sp} C$ is simply connected and the category of all those hyperalgebras $J$ of finite type such that $J^{\text {ab }}$ is finite-dimensional, and $J$ is proper. We see from Remark $4.12(1)$ that $(C, W) \mapsto\left(\left(C^{\circ}\right)^{1}, W^{*}\right)$ and 
$(J, V) \mapsto\left(J^{\circ}, V^{*}\right)$ give an anti-equivalence between the category of all those objects in cHCP such that $\mathrm{Sp} C$ is simply connected and the category of all those objects $(J, V)$ in iDHCP such that $J$ is of finite-type and proper, and $J^{\mathrm{ab}}$ is finite-dimensional. This, combined with Theorems 4.23, 3.9 (2), Proposition 6.2 and Lemma 6.4 proves the desired result. In fact, we see from Proposition 5.2 that $H \mapsto \mathrm{SSp} H^{\circ}$ gives a quasi-inverse of $G \mapsto$ hy $G$.

\section{UNIPOTENT AFFINE SUPERGROUP SCHEMES}

Proposition 7.1. Let $(C, W) \in \mathrm{HCP}$, and set $A=A(C, W)$ in AHSA. If there exists a non-zero element $w \in W$ on which $C$ trivially coacts, that is, $w \mapsto w \otimes 1$ via the $C$-comodule structure $W \rightarrow W \otimes C$, then A contains a non-zero odd primitive. In particular, if $C$ is irreducible and if $W \neq 0$, the conclusion holds true.

Proof. Let $(J, V)=\left(C^{\circ}, W^{*}\right)$ be the object in DHCP associated with $(C, W)$. For an element $w \in W$ as above, let $U$ be the subspace of $V$ consisting of those elements which annihilate $w$. The one sees that $U$ is a $J$ submodule of $V$, so that we have a sub-object $(J, U)$ of $(J, V)$. One sees that if $a \in J, v \in V$, then $\langle v \triangleleft a, w\rangle=\varepsilon(a)\langle v, w\rangle$, and so $v \triangleleft a-\varepsilon(a) v \in U$. By Proposition 5.5. $H(J, U)$ is normal in $H(J, V)$, and the corresponding quotient Hopf superalgebra $H(J, V) / / H(J, U)$ is isomorphic to $\wedge(\mathbb{k} x)$, which is generated by a non-zero odd primitive $x$ with $x^{2}=0$. Those elements in $A$ which annihilate, with respect to the pairing (4.17), the Hopf superideal $I:=H(J, V) H(J, U)^{+}$form a Hopf super-subalgebra, say $B$, which is naturally embedded into $(\wedge(\mathbb{k} x))^{*}$. We claim that this last embedding is an isomorphism; this obviously implies the desired result. Choose a totally ordered basis $Y$ of $U$, and add to it a final element, say $z$, to obtain a totally ordered basis, $X=Y \cup\{z\}$, of $V$. Let $\phi_{X}: J \otimes \wedge(V) \stackrel{\simeq}{\longrightarrow} H(J, V)$ be the isomorphism as given in Proposition 3.12. With [15, Remark 3.8] applied to $H(J, V) \rightarrow H(J, V) / I$, we see that $\phi_{X}^{-1}(I)=(J \otimes \wedge(U))^{+} \otimes \wedge(\mathbb{k} z)$; this, together with Proposition 4.22(2) (see its proof), proves the claim.

Recall from Definition 2.9(1) the definition of unipotent affine supergroup schemes.

Lemma 7.2. Given a short exact sequence $N \rightarrow G \rightarrow F$ of affine supergroup schemes, $G$ is unipotent if and only if $N$ and $F$ are both unipotent.

Proof. The 'only if' part follows since if the Hopf superalgebra $\mathcal{O}(G)$ which represents $G$ is irreducible, then $\mathcal{O}(F)$ and $\mathcal{O}(N)$, being its sub- and quotient coalgebras, are irreducible.

To prove the 'if' part, let $V$ be a simple rational $G$-supermodule. The $N$-invariants $V^{N}$ in $V$ form a $G$-super-submodule, since $N \triangleleft G$. If $N$ is unipotent, then $V^{N}$ is non-zero, and coincides with $V$ by the simplicity. Therefore, $V$ may be regarded as a simple rational $F$-supermodule, and is trivial as an $F$ - and $G$-supermodule if $F$ is unipotent.

The following theorem is due to Alexandr Zubkov, who informed privately the author of his proof. Here we give a simpler proof. 
Theorem 7.3 (A. Zubkov). An affine supergroup scheme $G$ is unipotent if and only if the associated affine group scheme $G_{\mathrm{res}}$ is unipotent.

Proof. Suppose $G=\operatorname{SSp} A$. Recall $G_{\text {res }}=\operatorname{Sp} \bar{A}$. Then the 'only if' part follows since if $A$ is irreducible, then so is the quotient coalgebra $\bar{A}$.

To prove the 'if' part, recall that $A$ is a union $\bigcup_{\alpha} A_{\alpha}$ of affine Hopf super-subalgebras $A_{\alpha}$. Since $\bar{A}=\bigcup_{\alpha} \bar{A}_{\alpha}$ by [15, Proposition 4.6(3)], we have only to prove, and will prove by induction on $\operatorname{dim} W^{A}$, that an affine Hopf superalgebra $A$ is irreducible, assuming that $\bar{A}$ is irreducible. We may suppose that $A$ is not purely even. Then by Proposition 7.1, $A$ contains a non-zero odd primitive, say $x$. Set $B=A /(x)$. Then we have a short exact sequence, $\wedge(\mathbb{k} x) \rightarrow A \rightarrow B$, of Hopf superalgebras, whence $\bar{A}=\bar{B}$, $\operatorname{dim} W^{A}-1=\operatorname{dim} W^{B}$ by Proposition 5.8(3). The induction hypothesis shows that $B$ is irreducible. This, together with the previous lemma applied to $\operatorname{SSp} B \rightarrow G \rightarrow \operatorname{SSp}(\wedge(\mathbb{k} x))$, proves the desired result.

\section{LineARLY REDUCTIVE AFFINE SUPERGROUP SCHEMES IN POSITIVE CHARACTERISTIC}

Lemma 8.1. An irreducible Hopf superalgebra $H$ is purely even if the Lie superalgebra $P(H)$ of primitives in $H$ is purely even.

Proof. Suppose that $H$ is a Hopf superalgebra. One sees from the wedge product construction given in [19, Sect. 9.1] that the subcoalgebras which appear in the coradical filtration of $H$ are super-subcoalgebras. If $H$ is irreducible, then we see as proving [19, Theorem 9.2.2] that the associated graded coalgebra $H^{\text {gr }}$ is an irreducible Hopf superalgebra. Therefore, by replacing $H$ with $H^{\text {gr }}$, we may suppose that $H$ is strictly graded in the sense of [19, Definition on Page 232] that $H(0)=\mathbb{k}, H(1)=P(H)$. By [19, Lemma 11.2.1], this implies that for every $n>1$, the $\mathbb{Z}_{2}$-graded linear map

$$
H(n) \rightarrow P(H)^{\otimes n}
$$

obtained by composing the $n-1$ times iterated coproduct $\left.\Delta_{n-1}\right|_{H(n)}$ : $H(n) \rightarrow H^{\otimes n}$ restricted to $H(n)$, with the projection $H^{\otimes n} \rightarrow H(1)^{\otimes n}=$ $P(H)^{\otimes n}$, is an injection. Therefore, if $P(H)$ is even, then every $H(n)$, and hence the whole $H$ are purely even.

The lemma just proven enables us to extend [16, Theorem 0.1] to the super situation, as follows.

Corollary 8.2. For an irreducible Hopf superalgebra $H$ of finite dimension in positive characteristic $p>2$, the following are equivalent:

(i) $H$ is semisimple as an algebra;

(ii) $H$ is purely even, and commutative semisimple as an algebra;

(iii) the base extension $H \otimes \overline{\mathbb{k}}$ to the algebraic closure $\overline{\mathbb{k}}$ of $\mathbb{k}$ is isomorphic to the function algebra $\overline{\mathbb{K}}^{G}$ of some finite p-group $G$;

(iv) the Lie superalgebra $P(H)$ is purely even, and is a torus in the sense that $P(H)$ is abelian as a Lie algebra, and every element in $P(H)$ generates a semisimple subalgebra in $H$. 
Proof. Obviously, (ii) $\Rightarrow$ (i). It is easy to see (ii) $\Leftrightarrow$ (iii). We see (iii) $\Rightarrow$ (iv), (iv) $\Rightarrow$ (ii), from the same implications of [16, Theorem 0.1], combined with Lemma 8.1

To see (i) $\Rightarrow$ (ii), it suffices, by Lemma 8.1, to prove that if $H$ is semisimple, it cannot contain a non-zero odd primitive. To the contrary, suppose that $H$ contains such a primitive, say $x$. Let $K$ be the Hopf super-subalgebra of $H$ generated by $x$. Then, $x^{2}$ is an even primitive in $K$, and the quotient Hopf superalgebra $K /\left(x^{2}\right)$ divided by the Hopf super-ideal generated by $x^{2}$ is isomorphic to $\wedge(\mathbb{k} x)$.

As a general fact we have that given a finite-dimensional semisimple Hopf superalgebra, every Hopf super-subalgebra, as well as every quotient Hopf superalgebra, is semisimple. This follows, for example, from (2.10), and from the corresponding result in the non-super situation (see [18, Corollary 3.2.3]), with the bosonization technique [17, Sect. 10] applied. But, this general fact implies that the Hopf superalgebra $\wedge(\mathbb{k} x)$ above is semisimple, which is absurd.

Recall from Definition 2.9 (2) the definition of linearly reductive affine supergroup schemes.

Lemma 8.3. A closed normal super-subgroup of a linearly reductive affine supergroup scheme is linearly reductive.

Proof. Just as in the non-super situation, it is known a Hopf superalgebra, say $A$, is cosemisimple if and only if the purely even, trivial $A$-comodule $\mathbb{k}$ is injective. By [15, Theorem 5.9(2)], given a conormal quotient $A \rightarrow D$ of a super-commutative Hopf superalgebra $A, A$ is injective as a left and right $D$-comodule. It follows that if $A$ is cosemisimple, then $D$ is, too. This is translated into the statement of the corollary.

Weissauer [27, Theorem 6] determined the form of linearly reductive algebraic affine supergroup schemes over an algebraically closed field of characteristic zero. According to his result, those supergroup schemes are rather restricted. In positive characteristic they are even more so, as is seen from the following.

Theorem 8.4. Assume char $\mathbb{k}>2$, and let $G=\operatorname{SSp} A$ be a linearly reductive affine supergroup scheme. Then $G$ is necessarily purely even. It follows by Nagata's Theorem (see [10, Chap. IV, Sect. 3, 3.6]) that if $G$ is algebraic and connected in addition, then it is of multiplicative type in the sense that the Hopf algebra $A$ is spanned by grouplikes after the base extension to the algebraic closure $\overline{\mathbb{k}}$ of $\mathbb{k}$; in particular, $G$ is abelian.

Proof. We prove only the first assertion, which implies immediately the second. Let $p=\operatorname{char} \mathbb{k}(>2)$. We may suppose that $A$ is affine, since it is directed union of affine Hopf super-subalgebras, which are necessarily cosemisimple. We may also suppose by base extension that $\mathbb{k}$ is algebraically closed. Then the Frobenius morphism gives rise to a short exact sequence

$$
G_{1} \rightarrow G \rightarrow G^{(1)}
$$

of affine supergroup schemes. Since $G^{(1)}$, represented by $A^{(1)}:=\left\{a^{p} \mid a \in\right.$ $A\}$, is purely even, it suffices, in virtue of Proposition 5.8(3), to show that 
$G_{1}$ is purely even. Note that $G_{1}$ is represented by the Hopf superalgebra $A_{1}:=A / I$, where $I$ is the Hopf super-ideal of $A$ generated by all $a^{p}$, where $a \in A_{0}^{+}$, and so that an isomorphism $A \stackrel{\simeq}{\longrightarrow} \bar{A} \otimes \wedge\left(W^{A}\right)$ such as given in Proposition 4.15 induces a counit-preserving superalgebra isomorphism $A_{1} \stackrel{\simeq}{\longrightarrow} \bar{A} / \bar{I} \otimes \wedge\left(W^{A}\right)$, where $\bar{I}$ is the ideal of $\bar{A}$ generated by all $a^{p}$, where $a \in \bar{A}^{+}$. The affinity of $A$ implies that $A_{1}$ is finite-dimensional and local, having $A_{1}^{+}$as a unique maximal ideal. Hence $A_{1}^{*}$ is irreducible. By Lemma 8.3. $A_{1}$ is cosemisimple, whence $A_{1}^{*}$ is semisimple. Therefore we can apply Corollary 8.2 to $A_{1}^{*}$ to conclude that $G_{1}$ is purely even, as desired.

\section{ACKNOWLEDGMENTS}

The work was supported by Grant-in-Aid for Scientific Research (C) 23540039, Japan Society of the Promotion of Science. The author thanks Alexandr Zubkov for his valuable comments on an earlier version of this paper. The author also thanks the referees for their very helpful comments and useful suggestions.

\section{REFERENCES}

[1] N. Andruskiewitsch, M. Graña, Braided Hopf algebras over non-abelian finite groups, Bol. Acad. Ciencias 63(1999), 45-78.

[2] G. Bergman, The diamond lemma for ring theory, Adv. in Math. 29(1978), 178-218.

[3] F. A. Berezin, Introduction to superanalysis, D. Reidel Publishing Company, Holland, 1989.

[4] J. Brundan, A. Kleshchev, Modular representations of the supergroup $Q(n) I$, Special issue celebrating the 80th birthday of Robert Steinberg, J. Algebra 260(2003), 64-98.

[5] H. Boseck, Affine Lie supergroups, Math. Nachr. 143(1989), 303-327.

[6] H. Boseck, Classical Lie supergroups, Math. Nachr. 148(1990), 81-115.

[7] C. Carmeli, L. Caston, R. Fioresi, Mathematical foundations of supersymmetry, EMS Series of Lectures in Mathematics, European Math. Soc., Zürich, 2011.

[8] C. Carmeli, R. Fioresi, Super distributions, analytic and algebraic super HarishChandra pairs, preprint, arXiv:1106.1972

[9] P. Deligne, J.-W. Morgan, Notes on supersymmetry (following Joseph Bernstein), Quantum Fields and Strings: A Course for Mathematicians, Vols. 1, 2 (Princeton, NJ, 1996/1997), pp.41-97, Amer. Math. Soc., Providence, 1999.

[10] M. Demazure, P. Gabriel, Groupes algébriques, Tome I, Masson \& Cie, Paris; NothHolland, Amsterdam, 1970.

[11] G. Hochschild, Algebraic groups and Hopf algebras, Illinois J. Math. 14(1970), 52-65.

[12] B. Kostant, Graded manifolds, graded Lie theory, and prequantization, Lecture Notes in Mathematics 570, Springer-Verlag, Berlin-Heidelberg-New York, 1977, pp.177306.

[13] J.-L. Koszul, Graded manifolds and graded Lie algebras, Proceedings of the International Meeting on Geometry and Physics (Florence, 1982), pp.71-84, Pitagora, Bologna, 1982.

[14] Y. I. Manin, Gauge fields theory and complex geometry, translated by N. Koblits and J. R. King, Grundlehren der mathematischen Wissenschaften 289, Springer-Verlag, Berlin-Heidelberg-New York, 1988.

[15] A. Masuoka, The fundamental correspondences in super affine groups and super formal groups, J. Pure Appl. Algebra 202(2005), 284-312.

[16] A. Masuoka, Semisimplicity criteria for finite-dimensional irreducible Hopf algebras in positive characteristic, Proc. Amer. Math. Soc. 137(2009), 1925-1932.

[17] A. Masuoka, A. N. Zubkov, Quotient sheaves of algebraic supergroups are superschemes, J. Algebra 348(2011), 135-170.

[18] S. Montgomery, Hopf algebras and their actions on rings, Amer. Math. Soc., 1993. 
[19] M. E. Sweedler, Hopf algebras, W. A. Benjamin, Inc., New York, 1969.

[20] M. Takeuchi, Tangent coalgebras and hyperalgebras I, Japanese. J. of Math. 42(1974), $1-143$.

[21] M. Takeuchi, On coverings and hyperalgebras of affine algebraic groups, Trans. Amer. Math. Soc. 211(1975), 249-275.

[22] M. Takeuchi, On coverings and hyperalgebras of affine algebraic groups, III, Amer. J. Math. 103(1981), 181-202.

[23] M. Takeuchi, Topological coalgebras, J. Algebra 97(1985), 509-539.

[24] M. Takeuchi, Review on 15], MR2163412(2006e:16066), Math. Reviews, Amer. Math. Soc.

[25] V. S. Varadarajan, Supersymmetry for mathematicians: an introduction, Courant Lec. Notes in Math. 11, Courant Institute of Mathematical Sciences, New York; Amer. Math. Soc, Providence, 2004.

[26] E. G. Vishnyakova, On complex Lie supergroups and homogeneous split supermanifolds, Transform. Groups 16(2010), No.1, 265-285.

[27] R. Weissauer, Semisimple algebraic tensor categories, preprint, arXiv:0909.1793

[28] A. N. Zubkov, Affine quotients of supergroups, Transform. Groups 14(2009), No.3, $713-745$.

[29] A. N. Zubkov, On quotients of affine superschemes over finite supergroups, J. Algebra and its Appl. 10(2011), 391-408.

Akira Masuoka: Institute of Mathematics, University of Tsukuba, Ibaraki 305-8571, JAPAN

E-mail address: akira@math.tsukuba.ac.jp 